Les Township tours au KwaZulu-Natal (Afrique du Sud) : d'une réappropriation historique et identitaire à l'avènement d'un socio-tourisme

\title{
Fabrice Folio
}

\section{(2) OpenEdition}

1 Journals

\section{Édition électronique}

URL : http://journals.openedition.org/echogeo/12243

DOI : 10.4000/echogeo.12243

ISSN : 1963-1197

Éditeur

Pôle de recherche pour l'organisation et la diffusion de l'information géographique (CNRS UMR 8586)

Référence électronique

Fabrice Folio, «Les Township tours au KwaZulu-Natal (Afrique du Sud) : d'une réappropriation historique et identitaire à l'avènement d'un socio-tourisme », EchoGéo [En ligne], Sur le Vif, mis en ligne le 09 décembre 2010, consulté le 01 mai 2019. URL : http://journals.openedition.org/echogeo/12243 ; DOI : 10.4000/echogeo. 12243

Ce document a été généré automatiquement le 1 mai 2019.

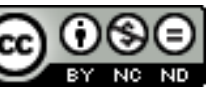

EchoGéo est mis à disposition selon les termes de la licence Creative Commons Attribution - Pas d'Utilisation Commerciale - Pas de Modification 4.0 International 


\title{
Les Township tours au KwaZulu-Natal (Afrique du Sud) : d'une réappropriation historique et identitaire à l'avènement d'un socio-tourisme
}

\author{
Fabrice Folio
}

La notoriété touristique de l'Afrique du Sud, en qualité de destination majeure sur le continent africain, est aujourd'hui reconnue. Le pays fonde son activité sur le niveau et la diversité de ses prestations, qui puise à la fois dans ses ressources naturelles et dans sa matière culturelle (Folio, Derroisne, 2010). De la nation arc-en-ciel, viennent initialement à l'esprit les parcs animaliers du Pilanesberg, du Kalahari Gemsbok ou du Kruger (aujourd'hui situé dans le Limpopo Transfrontier Park) ; sont aussi communément cités les sites impressionnants du Cap de Bonne Espérance, du Drakensberg et de Blyde Canyon. La richesse culturelle du pays, où s'identifient maintes ethnies bantoues, des marchés indiens, une route des vins dans «le coin des Français » et un carnaval Malais, confine pareillement à un bel éclectisme : terre de contacts, l'Afrique du Sud a généré, souvent en l'imposant, des identités multiples aux projections territoriales complexes, forgées au cours d'une histoire agitée (Gervais-Lambony, 2001). En ce domaine, des processus de réappropriation identitaire, à finalité économique, sont à l'œuvre à l'heure actuelle (Marschall, 2005). Un type de tourisme original, au caractère pédagogique et authentique, connaît un bel engouement: les Township tours. Encouragés aux divers échelons administratifs, ils composent un segment touristique (infra)-urbain sans égal. Qui plus est, ils se doublent d'une visée interne en réhabilitant des richesses communautaires longtemps spoliées. En somme, le processus assume un épisode médiatisé de l'histoire du pays, l'apartheid urbain, tout en tentant d'inverser, bon gré mal gré, l'image répulsive qui lui est associée. L'attirance pour les townships puise-t-elle dans une soif de connaissance pour la discrimination raciale institutionnalisée et la mise à bas d'une politique inique? Ou serait-ce plutôt la réputation de dangerosité, de pauvreté et « d'informalité » (Rolfes \& all, 2009), accolée à ces quartiers, qui agirait paradoxalement comme un produit d'appel ? 
2 Nous tenterons d'analyser le phénomène de mise en tourisme d'une prestation très spécifique, dont les enjeux n'ont d'égal que les appréciations tranchées qu'elle peut susciter. La controverse autour de cette attraction existe en effet, elle s'arrime au tourisme dit « sombre » ou « de pauvreté », qui laisse parfois place aux griefs portant sur un effet « zoo humain », voire sur un "voyeurisme à sensations ». Nous prendrons pour cadre de référence une province qui tient une place importante dans la construction historique du pays (Folio, 2007), à savoir le KwaZulu-Natal. Cette dernière-ci offre un potentiel de (re)découverte adapté à ce type de produit touristique. Il s'agira de voir comment cette province, confrontée aux ambiguïtés que sous-tend cette prestation et face à une concurrence rude de la part de sites placés sur le même créneau (tels que Soweto), tente aujourd'hui de se resituer en jouant d'une carte multiculturelle, connotée positivement et portée par des icônes.

\section{Afrique du Sud : une histoire torturée réappropriée à des fins touristiques}

\section{Tourisme, tourisme communautaire et devoir de mémoire}

3 Au préalable, il est intéressant de rappeler certaines statistiques touristiques concernant la nation arc-en-ciel. Elles insistent sur le poids prééminent de l'Afrique du Sud sur le continent africain, en contrepoint géographique de l'Afrique du nord et ses destinations égyptienne ou tunisienne. En 2008, le pays a accueilli 9,1 millions de visiteurs étrangers, soit une hausse de $8,3 \%$ par rapport à l'année précédente (SAT, 2008). Il importe cependant de rappeler que la majorité de ces touristes proviennent des pays limitrophes, gagnant l'aire de chalandise d'un Etat émergent (Lamy-Giner, Guebourg, 2005); les principaux voyageurs étrangers arpentant l'Afrique du Sud sont issus du Swaziland, du Lesotho ou du Zimbabwe. Toutefois, l'intérêt des touristes ultramarins pour ce pays est indéniable, en particulier les populations européennes (au sein desquelles les Britanniques, Allemands, Hollandais et Français), sans oublier les ressortissants Nordaméricains. Outre des liens historiques indéfectibles (pour Anglais et Néerlandais), ce tropisme s'appuie sur quelques atouts réels :

- Le riche patrimoine culturel et naturel de l'Afrique du Sud, terre de contrastes, vantée comme « le monde en un seul pays » (communication du South African Tourism).

- Le créneau habile sur lequel elle fonde son activité et qui hybride, avec un certain succès, le dépaysement africain aux facettes occidentales modernes et développées.

- Son rang de puissance économique africaine et le haut niveau d'infrastructures touristiques reposant sur un savoir-faire local, imputable en partie au legs britannique.

- Enfin et de façon plus paradoxale, la politique d'apartheid même (mais plus particulièrement son abrogation et son aboutissement démocratique) qui a fortement exposé le territoire aux regards du monde.

Le Livre blanc sur le tourisme (1996) et, au rang provincial le Livre blanc sur le tourisme au KwaZulu-Natal (2008), ont tous deux souligné les enjeux contemporains de l'investissement communautaire au sein de l'industrie touristique sud-africaine. $Y$ est notamment évoquée la nécessité de contribuer à la stratégie de rattrapage socio-spatial des populations discriminées par la politique de développement séparé. Pour bien le comprendre, rappelons simplement ici deux indicateurs : l'Afrique du Sud est un des Etats les plus inégalitaires au monde ; son coefficient de Gini atteint 0,578 , ce qui le situe entre 
le Brésil et la Colombie ou la Sierra Léone (United Nations Development Programme, 2007-2008). En dépit des politiques de discrimination positive (Affirmative Action), la ségrégation raciale historique a laissé place à une stratification socio-spatiale héritée en même temps que se complexifiant. Par ailleurs, les touristes internationaux contribuent à $8,1 \%$ du PIB national et dépensent 880 rands (soit environ $80 €$ ), par jour en moyenne, durant leur séjour en ce pays (SAT, 2008). Par voie de conséquence, une partie de l'équation recherchée par les décideurs consiste à faire en sorte que les Sud-Africains dans le besoin puissent au mieux accéder à une fraction de cette manne des devises extérieures.

En parallèle, l'étude de 2005 (DEAT) sur la compétitivité globale de l'industrie du tourisme en Afrique du Sud a souligné l'intérêt pour les attractions touristiques culturelles et héritées. La fascination (voire l'obsession) du pays pour tout ce concerne la célébration et la marchandisation de «l'héritage » (Marschall, 2005) peut se justifier par deux aspects : outre le fait que le tourisme soit devenu la panacée économique, il y a aussi la volonté d'instruire un ferment communautaire national. Le devoir de mémoire est clairement mis en avant, comme un sentiment de revanche sur l'histoire, allié à un vœu de rédemption et de projection vers l'avenir.

\section{Des bornes historiques}

6 Les Township tours, comme peut l'être également la découverte des villages traditionnels « ethniques " (Folio, 2010) ou encore la visite des musées d'apartheid, s'apparentent à autant de produits touristiques personnifiant, depuis quelques années, cette tendance à jouer sur la fibre culturelle, historique et identitaire. Ils émergent comme des niches d'une activité nationale se diversifiant. Spatialement, ils restent surtout représentés dans les principales provinces du pays (Gauteng, Western Cape, à degré moindre KwaZuluNatal et Eastern Cape). Ces attractions prennent appui sur la riche géohistoire nationale, parée de ses héritages san, bantou, boer/afrikaner, britannique ou indien. Elles sont en effet légitimées par un arrière-plan historique dense: le passé sud-africain est tumultueux et a été abondamment relayé par les grands médias internationaux. En conséquence, aux yeux des visiteurs, ces segments ont vocation à incarner une sorte de miroir des hauts-faits et des bifurcations historiques de l'Afrique du Sud, qu'ils soient précoloniaux, coloniaux, d'apartheid ou post-apartheid. Un pan de l'histoire du pays est particulièrement restitué : l'apartheid.

7 Cette période se caractérise, de façon générale dans le pays, en premier lieu par la promulgation, en 1948, de la politique ségrégative à l'encontre des non-Blancs (qui, il faut le rappeler, forme un prolongement uniforme d'un panel de lois discriminantes apparues depuis le début du siècle). La lutte qui lui fut associée, sans oublier la litanie de violences qui prit effet à l'aube des élections générales de 1994 (au KwaZulu-Natal comme dans la région de Johannesburg) marqua l'opinion internationale. Les stigmates de l'apartheid se lisent encore aujourd'hui dans l'espace urbain; ils ont pour cadre territorial les «townships" noirs (ces ex-quartiers ouvriers implantés en troisième couronne des villes), ainsi que les aires d'habitat informel qui les interpénètrent et/ou les entourent (Folio, 2004). Ceux-ci matérialisent les lieux célèbres de la désobéissance civile et, par certains aspects, d'une violence, à tout le moins vue de l'extérieur, comme presque légitime ${ }^{1}$. 
Cette phase historique, centrée sur les populations de couleur (en particulier noires), est depuis une quinzaine d'années associée au projet national de la diversification touristique à usage interne et externe. Plus largement, lorsque l'on parle de formes de tourisme hérité et ethnique (pour ne pas dire racial) en Afrique du Sud, il faut d'abord avoir à l'esprit que ces prestations s'inscrivent dans l'émergence récente et universelle de segments liés à de nouvelles attentes - la quête de l'unique, du différent -, qu'ils soient responsables, d'aventure, soutenables ou de mieux-être (Poon, 1993). Pour le pays, il faut ensuite prendre en considération le désir de vendre une histoire nationale connue et (ré)assumée, induisant un tourisme quasi-cathartique et décomplexé. Sur le terrain, cela prend concrètement la forme de circuits urbains dans les townships ou encore de visites de villages, par exemple zoulous, en monde rural (Dondolo, 2001). Tout deux répondent à des bornes historiques séquencées (l'apartheid et antérieurement la période coloniale). A cela, nous ajouterons l'accent mis sur les musées dits d'apartheid (KwaMuhle dans la province), lesquels sont venus étoffer une offre muséologique ancienne (musées d'histoire naturelle, d'histoire locale...), en tentant de la renouveler par une expérience plus affective. Dés lors que les motivations des visiteurs (surtout étrangers, comme on pourra le voir) s'orientent sur le regard qu'ils portent sur d'autres peuples et le souci de compréhension de ce qui leur est dissemblable (l'Autre), ces prestations émargent inévitablement au secteur du tourisme culturel (Ramchander, 2004).

Illustration 1 - Province du KwaZulu-Natal, tourisme historique et identitaire

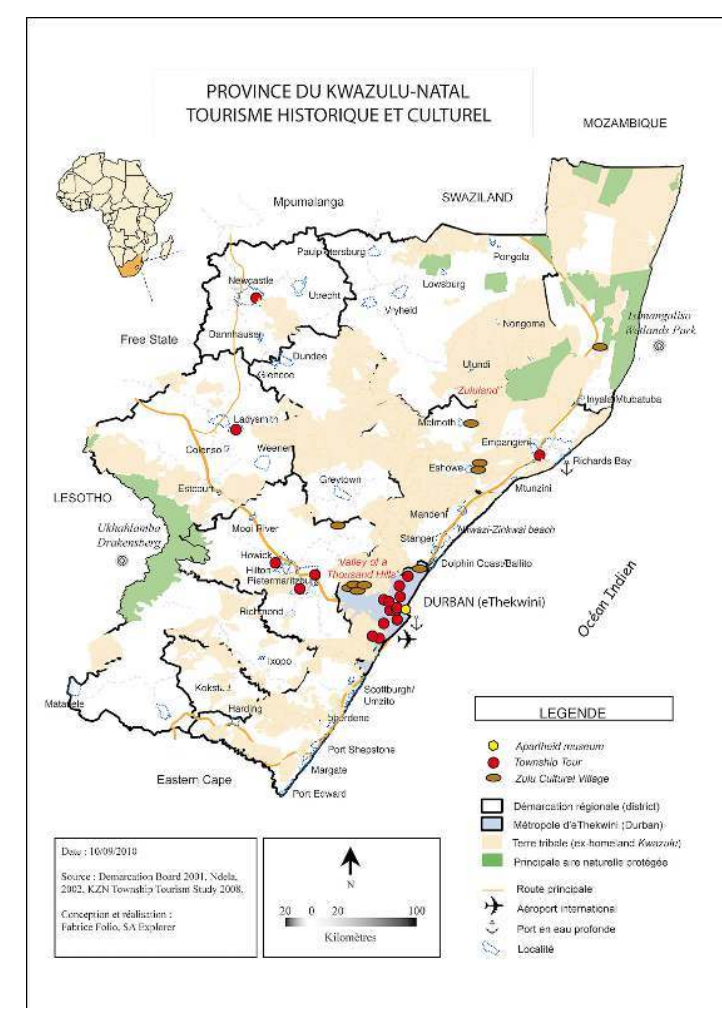

Sources : Demarcation Board 2001 ; Ndela, 2002 ; KZN Township Tourism Study 2008 - conception et réalisation : Fabrice Folio 


\section{Aperçu d'une nouvelle expérience touristique : une clientèle et une géographie évidente?}

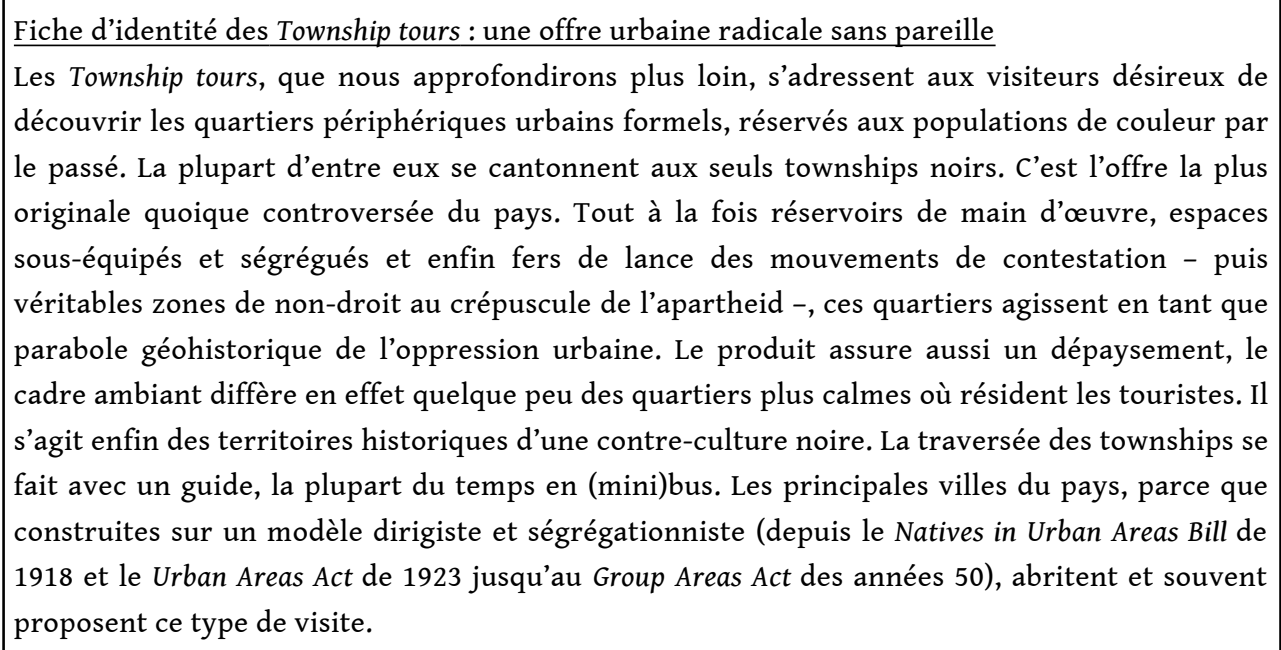

9 Sur le papier, cette prestation s'apparente, d'une part, à un tourisme culturel reposant sur la composante majoritaire de la population sud-africaine. D'autre part, elle met en exergue des espaces et/ou des communautés ayant enduré les affres de la politique de développement séparé. Le produit occupe une place qui gagne en importance dans l'activité économique nationale. En dehors d'une clientèle domestique spécifique (en général dans le cadre des sorties scolaires), il est malgré tout l'apanage d'une population étrangère et ultra-marine ${ }^{2}$. Les townships ont à l'origine incarné des espaces cibles pour la propagande du régime raciste, puis à l'inverse pour les activistes anti-apartheid, les bailleurs étrangers et les aménageurs. Ce n'est que récemment que le touriste étranger a commencé à s'y intéresser mais les Sud-Africains, notamment blancs, y sont toujours restés à l'écart (de même que les touristes Africains, Chapman, 2003). Cela demeure un phénomène intéressant à approfondir mais les touristes domestiques, issus de la province même ou des hautes terres du pays, restent pour l'essentiel attachés à la facette récréative littorale du KwaZulu-Natal. Dans la communication marketing diffusée dans les brochures touristiques ou sur les sites Internet des prestataires du Township tour, le touriste « hyper-occidental » (Allemand, Dagorn, Vilaça, 2005), en particulier européen et nord-américain, semble prioritairement interpellé. Il faut le lire par le fait que cette prestation renvoie à ces ressortissants ultra-marins une page d'histoire illustre, un " exotisme » africain séculaire (Staszak, 2008, Tomaselli, 2001) et ainsi parfois un écho déformé d'une mémoire civilisationnelle en prise avec le continent noir. Evidemment, ce ne sont pas là les seuls motifs à retenir, le caractère multi-sensoriel et novateur de ces tours entrant sans conteste en ligne de compte. Toutefois, cela compose un élément de positionnement, « occidento-centré », non négligeable bien que pour le moins inavoué.

10 La lecture spatiale urbaine, a priori évidente quant à leur localisation, est de surcroît plus complexe qu'il n'y paraît (illustration 1). Par exemple, en tant qu'autre attraction culturelle historique, la situation des musées d'apartheid reste assez classique (position centrale ou péricentrale dans les CBD des grandes villes). De même, la localisation des Cultural villages traditionnels pousse à évoquer spontanément la ruralité. Encore qu'ici, cette prestation vise une clientèle touristique basée en ville et a subséquemment opté 
pour une implantation en zones villageoises non loin des espaces urbains (ou des grands axes routiers), soit une situation plutôt semi-rurale. Pour sa part, la position géographique des Township tours concerne les ex-quartiers africains des agglomérations. Cependant, il faudrait plutôt parler d'une localisation périphérique extrême, où les relations avec les zones villageoises via les aires informelles périurbaines n'ont que peu cessé. Au contraire, l'éloignement relatif du cœur des villes, en dépit de liens fonctionnels, incite à proposer le terme d'infra-urbain. Au final, il en ressort, pour la totalité de ces prestations vues en commun, une disposition en gradient centrepériphérie (espace central, quartier urbain lointain et zone semi-rurale).

\section{Les Township tours du KwaZulu-Natal, produit pédagogique ou sensationnel ? Les ambiguiités de la mise en tourisme}

\section{Déroulement du tour : des espaces touristiques convoités et appréhendés}

11 Le KwaZulu-Natal est la province la plus peuplée d'Afrique du Sud. Il se situe au nord-est $\mathrm{du}$ pays en position frontalière avec trois autres pays (Lesotho, Swaziland et Mozambique). La région a accueilli 1,4 millions de visiteurs étrangers l'année 2008, ce qui fait d'elle la troisième destination nationale (SAT, 2008). Toutefois, sa clientèle s'avère essentiellement domestique: outre les provinciaux mêmes, il s'agit de la première destination des vacanciers sud-africains en provenance des hautes terres centrales, surtout du cœur économique, le Gauteng. Son potentiel réside, à l'image du pays, sur des caractéristiques à la fois paysagères et culturelles. On peut évoquer un liseré côtier constitué de dunes, des plages de sable blanc au devant d'une eau réchauffée par le courant des Agulhas, des lagunes étendues (Ste Lucia), des contreforts montagneux (le Drakensberg), pléthores de réserves animalières (Hluhluwe, uMkuze...) et enfin les cultures zouloue et indienne. Deux de ses sites (Isimangaliso Wetland Park et uKhahlambaDrakensberg Park) sont classés au patrimoine mondial de l'humanité.

Les excursions organisées à l'intérieur des townships sont au nombre de 17 dans la province (KZN Township Tourism Study, 2008). Elles sont rattachées prioritairement aux quartiers périphériques des grandes villes, au premier chef d'entre-elles Durban/ eThekwini qui en regroupe la majorité (10 expériences recensées). La métropole économique est suivie de la capitale administrative, Pietermaritzburg, à $80 \mathrm{~km}$ dans l'arrière-pays proche, ainsi que des villes secondaires de Richards Bay, de Ladysmith ou de Newcastle. Ces dernières se situent plus au nord et dans l'intérieur des terres, le long des Nationales 2 et 3, soit des colonnes vertébrales, en forme de T inversé, de la province (illustration 1). 
Illustration $2 a$ - Les maisonnettes locatives construites en série sous l'apartheid dans le township de Sobantu (Pietermaritzburg)

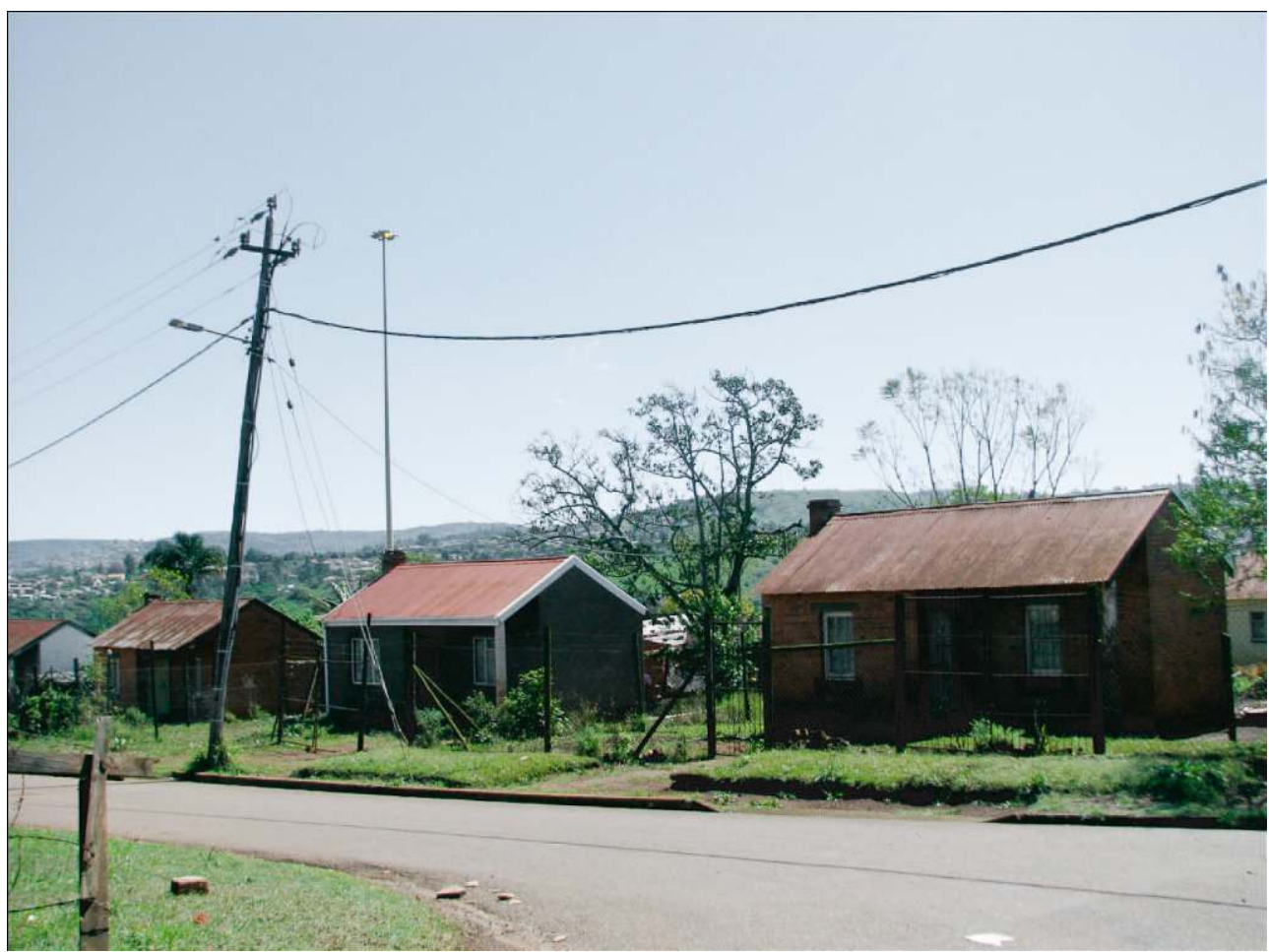

Auteur : Fabrice Folio.

Illustration 2b - Des enfants s'animant et se regroupant lors de l'arrivée des touristes à Cato Manor, Durban

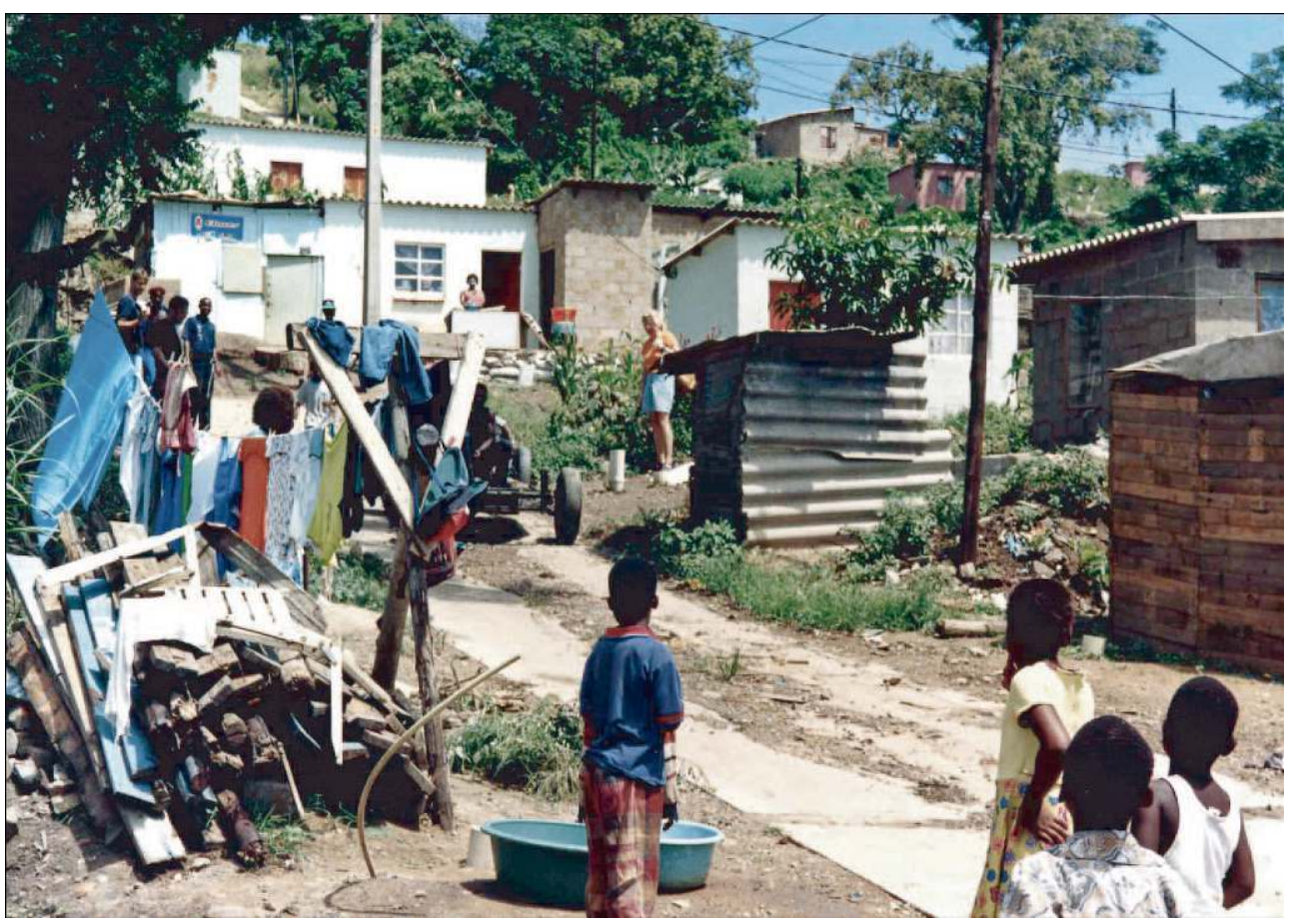

Auteur : Fabrice Folio. 
Illustration 2c - Un spaza shop, boutique de produits de première nécessité, au sein du township d'eSikhawini à Richards Bay

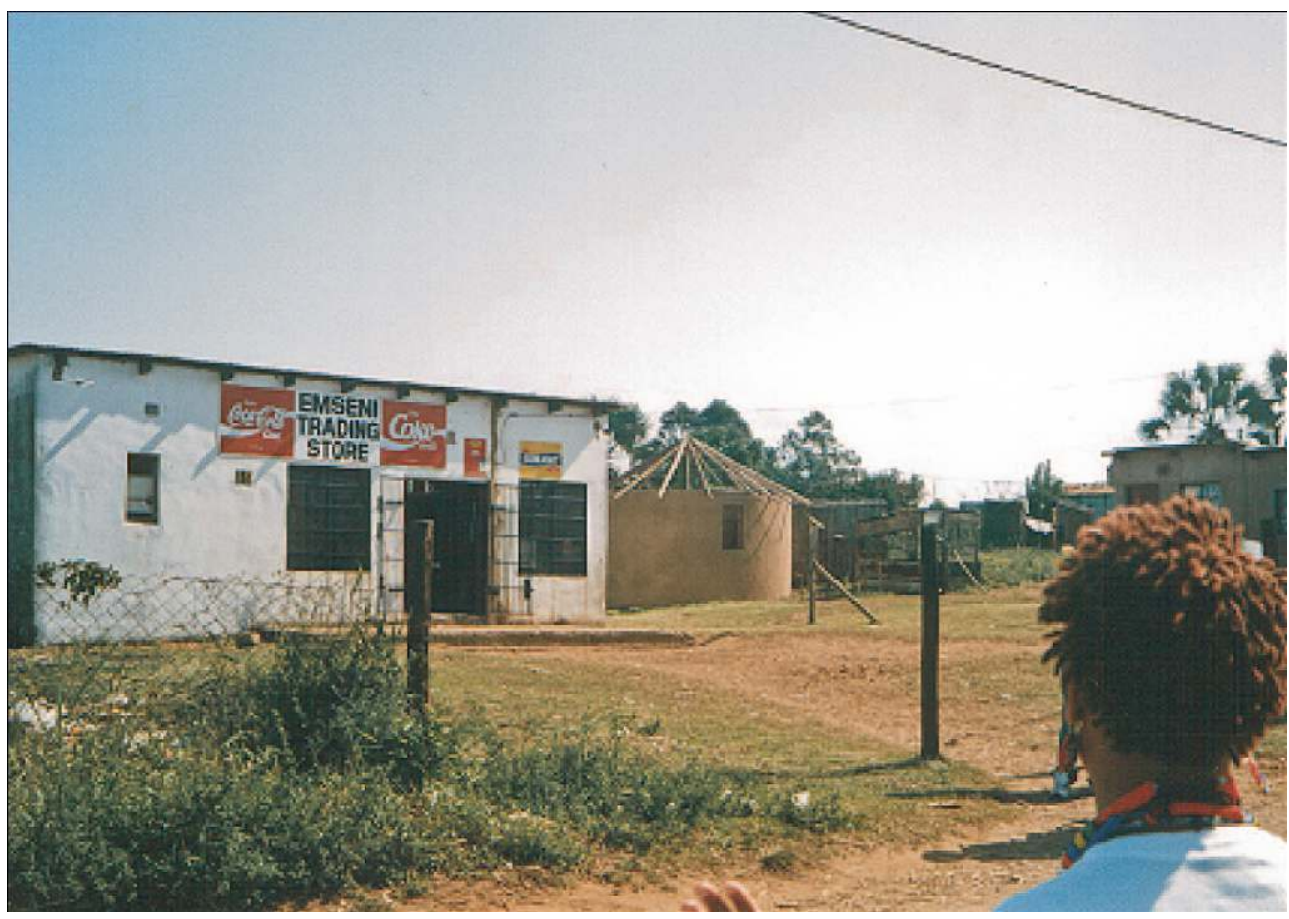

Auteur : Fabrice Folio.

Il est possible de retracer, dans ses grandes lignes, le déroulement du tour. Celui-ci s'exécute généralement sur une demi-journée (3-4 heures), plus rarement à la journée. Le touriste se voit facturer entre 250 et 450 rands ( 25 à 45 euros). Il prend place au sein d'un minibus (avec un maximum de 6 à 8 personnes) voire, pour les structures touristiques les plus modestes, au sein d'un véhicule individuel qui peut parfois être le sien. Pour les expériences durbanites, le départ se fait depuis le musée KwaMuhle, avec une entrée en matière théorique. Autrement, il a lieu depuis l'office du tourisme ou depuis l'hébergement du visiteur.

La traversée du township se "déroule " par la suite. En règle générale, c'est une expérience motorisée, avec en toile de fond les explications transmises par le guidechauffeur. Il s'agit fréquemment d'une personne originaire ou résidant dans le quartier. La réputation de dangerosité de ces espaces n'est pas écartée, des consignes de sécurité pouvant être données (ne pas s'éloigner, garder les vitres fermées en certains endroits...). Le visiteur n'est de toute manière pas libre de déplacer où il le souhaite et suit les instructions de l'accompagnateur.

Le paysage caractéristique de l'ex-quartier ségrégué se met alors à défiler. Il est marqué avant tout par les alignements austères des ex-maisonnettes locatives «boites d'allumettes " (matchbox houses) et par la compacité ainsi que l'effervescence des lieux, dont on observe qu'elles marquent une rupture avec les autres quartiers urbains (illustrations 2). Aux alentours, les frontières historiques rigides des townships s'étiolent : définies comme espaces tampons (buffer zones), dont l'utilité initiale était de contrôler ces secteurs, les étendues vierges ont été colonisées par des poches d'habitat précaire et de nouveaux lotissements sociaux rectilignes. 
16 Des arrêts sont prévus, durant lesquels le visiteur est amené à ressentir l'atmosphère vibrante du township et à interagir avec les résidents: ce sont généralement des supérettes (spaza) qu'environnent des étals chamarrés de commerce informel, des tavernes ou sheebens (bars clandestins de vente d'alcool sans licence, bannie sous l'apartheid), parfois encore une école primaire ou un hostel (logement collectif ouvrier masculin), voire la traversée d'un camp de squatters et ses shacks (taudis) imbriqués. Enfin, un déjeuner peut être proposé sur la place centrale du township (braai ou barbecue), de temps à autre chez la famille du guide.

17 Ce déroulement semble quasi le même dans les townships provinciaux comme dans ceux d'autres villes du pays, ce qui laisse à penser que la prestation est de plus en plus formatée.

\section{Controverse autour du produit ; de quel type de tourisme parle-t-on exactement?}

18 Au plan national, les tours de township sont depuis quelques années placés sous le feu roulant des critiques. Incontestablement, c'est « l'effet zoo » (ou pourrait-on dire « l'effet réserve humaine » pour davantage rester dans le ton du pays) qui est mis en avant. Le malaise que ce type de tour peut entraîner, pour le touriste à l'abri des vitres fumées des minibus climatisés, comme pour l'habitant épié dans son quotidien, est fréquemment dénoncé - même si c'est parfois sur le ton du sarcasme -, à la fois dans les médias sudafricains et au plan académique (Butler, 1999, Holliger, 2005, cf. planche satyrique de Madam \& Eve, 2002 - illustration 3). Pour être plus précis, il est critiqué une condescendance étrangère à venir scruter la pauvreté d'un pays émergent dans le cadre d'un bien de consommation (Bester \& Buntman, 1999, Jeuwsen, 1996). D'autant que cette marchandisation culturelle est augmentée, pour l'Afrique du Sud, du ressort criminel, lequel induit sur place une excitation déplacée, à peine refoulée par la gravité du lieu. 


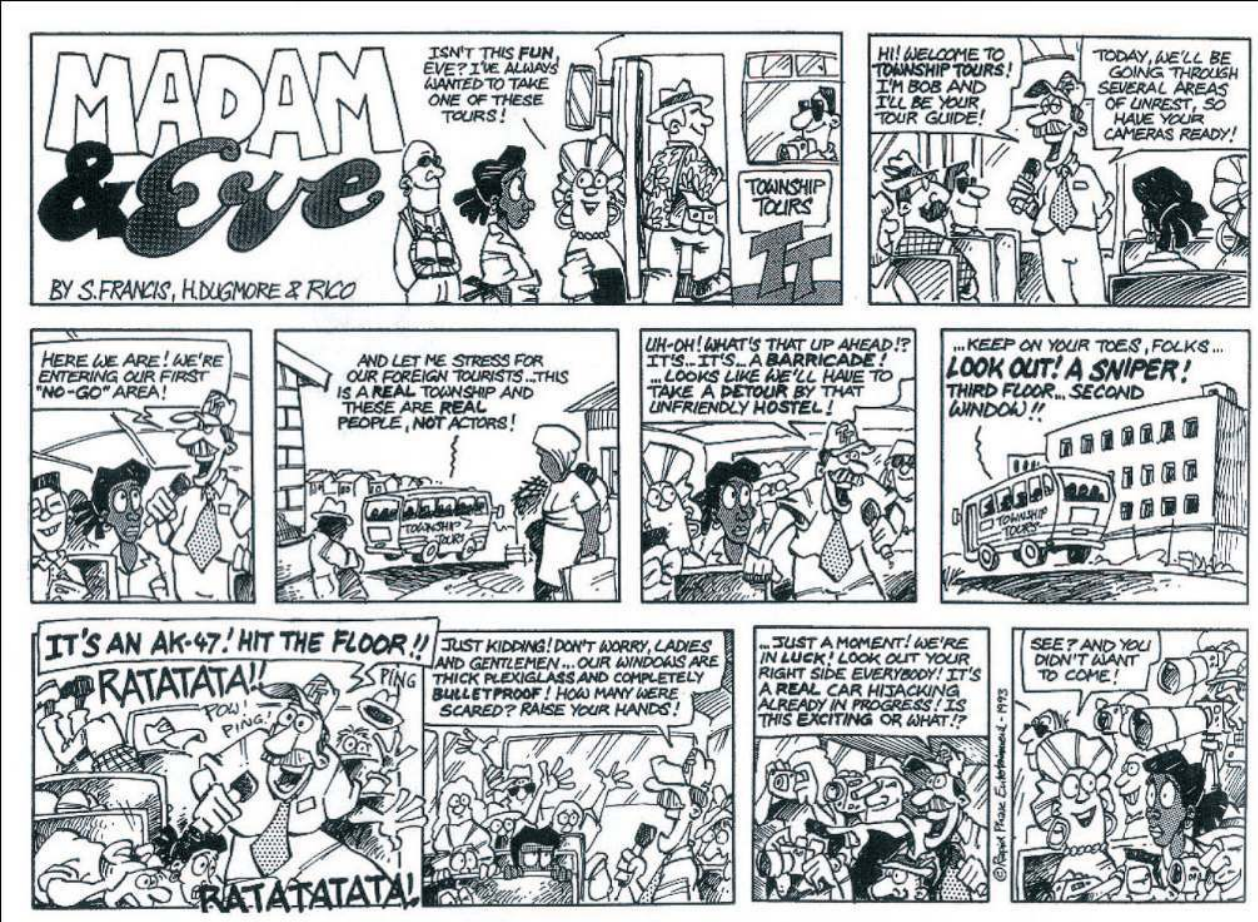

Publiée dans les grands journaux du pays, cette planche décrit de façon humoristique le folklore inhérent à certains Township tours. Le guide, tout en soulignant l'aspect « réel » de l'expérience, joue particulièrement sur le registre des émotions garanties en dépeignant la criminalité locale et plus largement le danger à être présent dans une telle zone de non-droit.

Source : S. Francis, H. Dugmore \& Rico, 2002 (publiée avec l'aimable autorisation des auteurs).

Les Township tours s'articulent en effet sur le couple admiration-répulsion pour populariser leur offre. Certes, cela n'est pas évoqué en ces termes, l'intérêt culturel est évidemment exhibé par les prestataires. Officiellement, ils visent à faire comprendre de façon tangible, en le lisant dans le paysage, ce qu'ont été et ce que sont encore les stigmates de l'apartheid urbain pour une majorité d'individus. Cette facette s'additionne à la possibilité de mesurer le chemin parcouru depuis 1994 (constructions matérielles et politiques de développement menées in situ, Masland \& al, 2002). Mais derrière cette immersion pédagogique, une fascination particulière se tisse autour de la visite de ces espaces reculés, peu visibles et à la réputation affirmée. Epicentres de la lutte contre l'apartheid, les townships gardent aujourd'hui une image persistante de quartiers dangereux en Afrique du Sud. Si cela demande à être relativiser (les CBD le sont tout autant et ce sont souvent des sections des townships qui sont concernées), il n'en reste pas moins que ce sont les espaces parmi les plus démunis, repaire du tsotsi (voyou), où chômage et activités interlopes sont très ancrés.

A la diatribe du voyeurisme déplacé, s'ajoute par conséquent celle exhumant un côté sensationnel incongru. De leur côté, les opérateurs mettent plutôt en avant la faculté qu'ont ces tours de combattre les clichés: les townships ne peuvent être réduits à des quartiers désœuvrés, malfamés, en marge de tout (Else \& al, 1997). Ils ne sont pas non plus des bidonvilles et d'autres groupes raciaux (ainsi que de multiples ethnies) y sont représentés. 
21 Si l'on revient sur les récriminations citées plus haut, celles-ci sont à replacer dans les balbutiements d'une réflexion herméneutique qui environne ce type de prestation. Les Township tours entrent en effet en résonance avec une amorce de théorisation du phénomène dans les médias généraux ou spécialisés, qui invite en tout cas à cesser de les considérer de façon isolée. Ainsi, le site Slate.fr a consacré en novembre 2009 un article à ces nouvelles formes de "tourisme de choc », énumérant des expériences similaires dans la favela de Rocinha à Rio, le bidonville de Dharavi à Mumbai ou sur un autre registre Tchernobyl en Ukraine ${ }^{3}$. Cette expression renvoie au "tourisme sombre " (dark tourism) évoqué par A. Kendle dans le journal Vagabondish, The Travelzine for today's vagabond (2008), bien que ce soit J. Lennon et M. Foley (2000) qui l'aient initialement portée sur les fonts baptismaux. La définition s'affine en ce que les Township tours (à l'instar des exemples brésilien, indien, mais aussi de la visite de zones d'immigrants à Rotterdam ou de quartiers difficiles de Houston) composent une sous-catégorie du «tourisme de pauvreté $»^{4}$. Leur principale caractéristique est d'être de plain pied dans le(s) «Sud» planétaire(s), ainsi que de se dérouler dans un espace de la normalité pour les habitants. A l'observation de certains de ces Etats (Brésil, Inde, Afrique du Sud...), on voit que les aires fréquentées sont souvent celles de pays en devenir détenant de très forts contrastes internes : l'opulence y tutoie la misère et on peut penser que c'est ce clivage interne, peut être même plus qu'un différentiel avec le lieu de départ du visiteur, qui va venir interpeller ce dernier. Par tourisme de (ou contre la) pauvreté, R. Scheyvens (2002b) y voit pour sa part, dans une visée plus humaniste, une forme de découverte de zones paupérisées qui adhère aux principes du tourisme alternatif: celui-ci promeut une démarche éthique et durable entre des protagonistes de rang extrême (compréhension mutuelle, redistribution locale des fruits de l'activité et égalité de traitement, Holden, 1984). L'objectif est d'opérer un rééquilibrage direct et responsable des richesses planétaires entre les plus nécessiteux et les plus solidaires, en se sentant citoyen du monde.

$\mathrm{Au}$ final, les tours de townships sont pour nous à l'interface de plusieurs segments touristiques et d'une double influence sous-jacente cohabitant (illustration 4): il s'agit d'un tourisme urbain (mais différent des city tours ponctuant les principales villes de la planète) et d'un tourisme culturel et ethnique (Ramchander, 2004), quoique se distinguant, par exemple, des circuits en villages traditionnels qui sont fréquemment des spectacles rodés. A ce stade, on comprend que les volets historiques et pédagogiques tiennent une place centrale. Le but est de comprendre l'apartheid urbain, sa genèse, sa nature et son évolution. Il s'agit ensuite d'un néo-segment touristique, axé sur l'interaction et la réalité (Freire-Medeiros, 2009), où le « refoulé » tient lieu de produit d'appel. Dans une acception critique, ce tourisme de pauvreté (on citera également l'anglicisme poorism), ne fait aucunement l'impasse sur la radicalité de l'approche (riche/ pauvre), le voyeurisme et la quête d'émotions fortes (par rapport à l'image mentale que nourrissent ces quartiers, Weiner, 2008, Gentleman, 2006). Dans une acception résolument engagée, il adhère, en théorie, au tourisme alternatif (bien qu'à nuancer d'un tourisme humanitaire par un aspect grand public). On parlera donc cette fois de tourisme communautaire ou " pro-pauvre » (Rogerson, 2006, Ashley \& al, 2001), qui consiste à se rapprocher de ceux dans le besoin, afin de comprendre et d'aider, tout en en retirant un enrichissement personnel (soit une « solidarité de contact ", en opposition par exemple à des donations envoyées à des $\mathrm{ONG}$ ). Quoi qu'il en soit, le registre émotionnel et sensationnel est, pour ces derniers aspects, davantage prononcé. Afin de tenter de 
considérer ce large spectre, nous soumettons le terme générique de " socio-tourisme ». Ce dernier fait écho à celui d'" éco-tourisme ", quant à la participation en petits groupes, aux logiques de respect, de sensibilisation et de redistribution, bien qu'il reste avant tout basé sur l'homme et son histoire.

Illustration 4 - Les Township Tours en Afrique du Sud : un socio-tourisme?

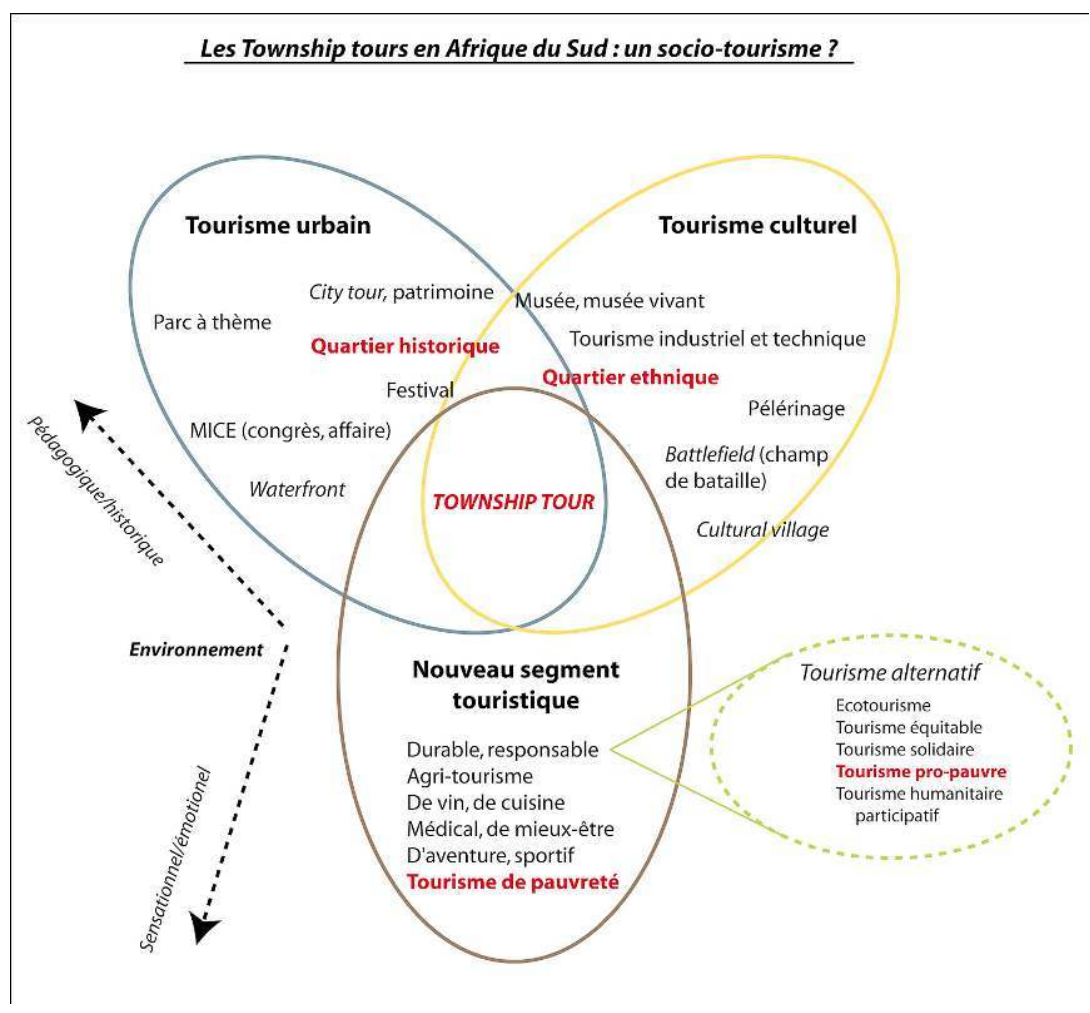

\section{Les Township tours et ses dénominateurs : authenticité, interaction, pédagogie}

Les Township tours n'échappent pas, on le voit depuis plusieurs années, à une réflexion critique. Toutefois, ce produit ambigu détient des ressorts assurément complexes et mérite de ce fait une analyse plus fine. Nous pouvons tenter de la conduire pour le KwaZulu-Natal en combinant trois critères. Ceux-ci sont autant de caractéristiques que ces prestations vantent : d'abord leur caractère authentique ; ensuite un rapport à l'autre sans pareil; enfin leur apport pédagogique. Quel est le degré de réalisme et la place de l'éthique dans le cadre de cette prestation? Qu'en est-il réellement de la nature des interactions avec les résidents? Enfin, quelle est la part d'enrichissement culturel qu'elle peut engendrer?

Contrairement aux Cultural villages zoulous qui sont des représentations reconstituant la vie d'autrefois (avec des acteurs recrutés et rémunérés), les Township tours se déroulent dans un lieu de vie réel, le seul acteur touristique étant a priori le guide-chauffeur. Dès lors, le visiteur est littéralement immergé, il a l'impression de toucher à une expérience unique, où les doutes quant à l'authenticité ne l'assaillent plus: il se situe tout simplement dans les principaux quartiers urbains sud-africains en taille humaine. Il reste que les circuits choisis par les opérateurs ne représentent qu'une portion assez infime du 
paysage de ces vastes espaces, nos visites répétées des townships d'Umlazi et de Kwamashu à Durban l'attestant. Ils peuvent de la sorte être identiques d'un tour à l'autre, mais aussi être fléchés et organisés, les locaux pouvant être avertis du passage d'étrangers, comme à Sobantu (Pietermaritzburg) ou à Clermont et Umlazi (Durban) : des réunions d'informations sont ainsi organisées en amont dans les centres communautaires avec les responsables locaux, afin de sensibiliser la population et optimiser autant que possible l'accueil 5 . Des recherches plus fines restent à conduire sur les réactions locales à ce type de tourisme - ce que S. C. Dubin (2006) a nommé the politics of reception - mais il convient déjà de relativiser quelque peu la part de découverte intégrale et de spontanéité exclusive associée à ces prestations.

Par rapport aux produits touristiques conventionnels, les Township tours font aussi état, souvent avec insistance, d'une interaction réelle avec les résidents. Force est de constater que celle-ci se résume à l'interlocuteur principal qu'est le guide. Le tour en minibus privatif crée un espace encapsulé (Butler 2003b), où la bulle touristique prend la forme d'un habitacle clos avec air aseptisé, coupé des éventuelles nuisances extérieures. Quant aux rencontres faites tout au long du tour, elles sont surtout marquées par la célèbre poignée de main sud-africaine au sein d'un bar, les échanges fugaces de courtoisie (durant les achats) et les contacts avec des groupes d'enfants dans un joyeux brouhaha de prises de photographies et de distribution de bonbons... Cette interaction même minimale a le mérite d'exister, bien que le rapport à l'autre puisse être parasité par un voyeurisme lancinant, aggravé par les appareils technologiques nomades. Les touristes sont effectivement invités à filmer et à prendre des photographies (une différence avec certains tours de Mumbai par exemple, où cela reste interdit); ceci, du moins, lorsque le champ est large, les portraits d'habitants étant tolérés sous réserve de règles de politesse élémentaire (mais dans les faits à la discrétion du visiteur). Les touristes sont d'ailleurs inégalement à l'aise avec cela. Rappelons ici que le fait de photographier et de documenter sur la pauvreté résulte de l'importation d'une tradition britannique de journalisme « des bas-fonds » datant du $19^{\mathrm{e}}$ siècle ; elle était le fait d'écrivains issus de la bourgeoisie, désireux de pénétrer en profondeur l'espace urbain (Stallybrass \& White, 1986).

L'assise culturelle est également palpable, le quartier étant présenté tel qu'il est, c'est à dire tel qu'il a pu évoluer sous l'apartheid ${ }^{6}$ et depuis son abrogation: mise en place d'équipements et de services publics, érection de logements sociaux, apparition de quartiers aisés et sécurisés (illustration 5$)^{7}$. Toutefois, y compris à travers les informations apportées par le guide, les Township tours nous paraissent hésiter entre la prestation ludique (reprenant les codes mondiaux du tourisme de loisir et destinée aussi à alléger, pour le confort du visiteur, la solennité des lieux) et l'excursion historicogéographique fouillée et didactique. Aussi proposent-ils en général un même produit hybride mêlant discussions légères et explications superficielles sur l'apartheid. Relativisons tout de même ce point par le fait que des données plus étayées et précises peuvent être délivrées par certains guides, en fonction des questions posées. Il demeure que même si le tour offre un fond narratif et explicatif, il continue la plupart du temps à reproduire certains stéréotypes (Perkins, 1979, Butler, 2003a). L'impression finale qu'il tend à laisser, bien que nuançant le caractère dangereux et miséreux des townships, est indéniablement celle d'un tiers-monde exotique, coloré et chaleureux; soit une vision essentialiste d'une culture "africaine» ici territorialisée. Les brochures mêmes véhiculent ces images de figures archétypales : individus fraternels et souriants, cadre 
bâti dégradé, commerce informel omniprésent... Le visiteur de son côté est placé dans la position du héros solidaire, sorte de grand témoin de l'ordre mondial inégalitaire et d'aventurier global empathique. Les donations et achats sont encouragés mais donnent presque l'impression de flatter un ego humanitaire plutôt que d'offrir une argumentation de fond sur la vie locale et les formes de mobilisation et de responsabilisation. Le tout peut ainsi pérenniser - tout en le dénonçant - un ordre social différencié et inégalitaire. Parce qu'il s'agit d'une prestation payante et qu'il est jugé que les visiteurs ne sont pas prêts à sacrifier une expérience divertissante et contrôlée sur l'autel du souci du détail et de l'imprévisible, la palette des disparités socio-économiques et du jeu politique interne est en partie passée sous silence.

Illustration 5a - Le township d'Umlazi

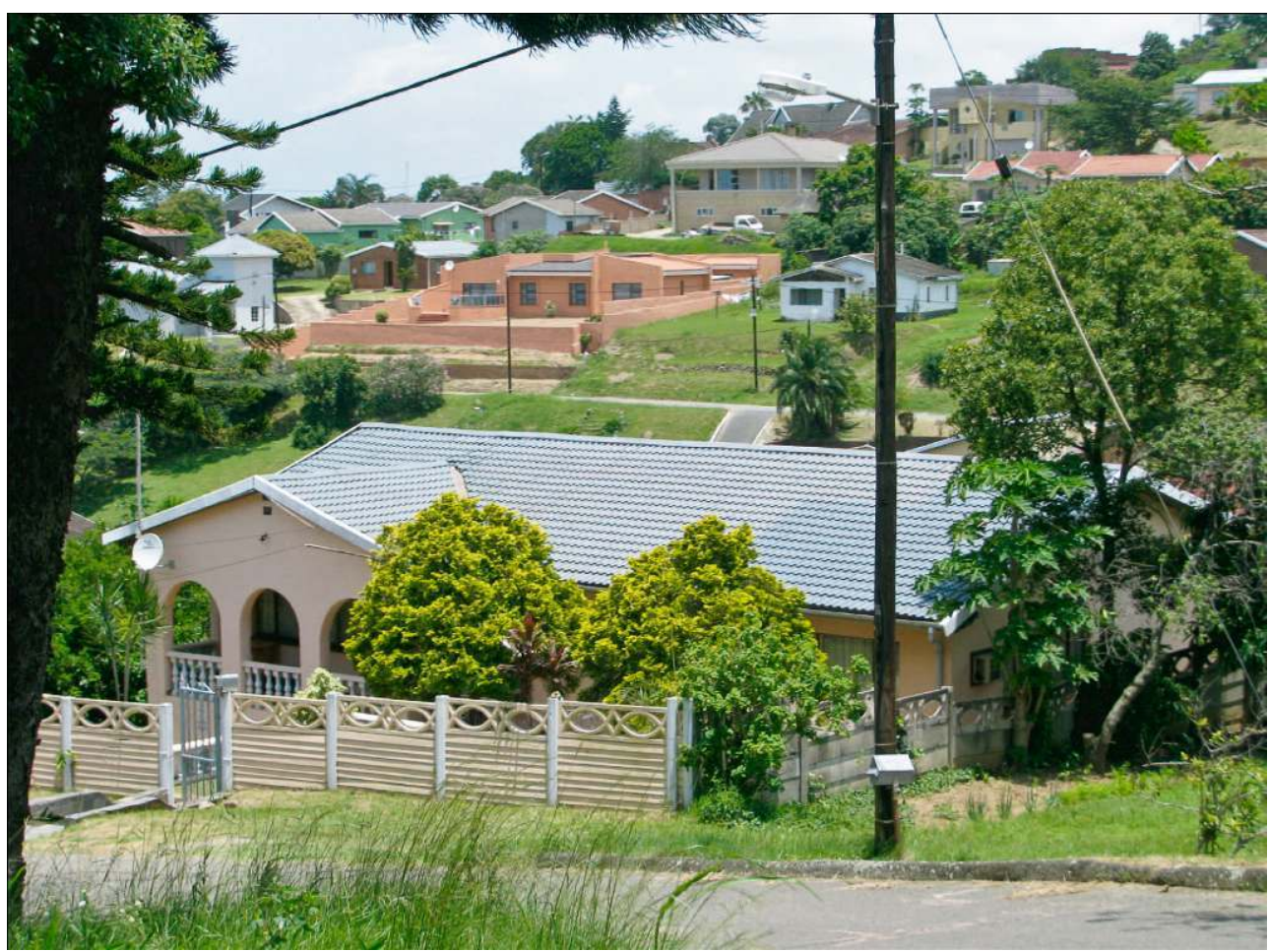

Peuplé d'environ 750000 habitants, il fait aujourd'hui l'objet d'investissements importants de la part de la municipalité d'eThekwini. Les contrastes, en partie hérités depuis sa création en 1967, se creusent ainsi avec l'apparition de quartiers aisés.

Auteur : Fabrice Folio. 


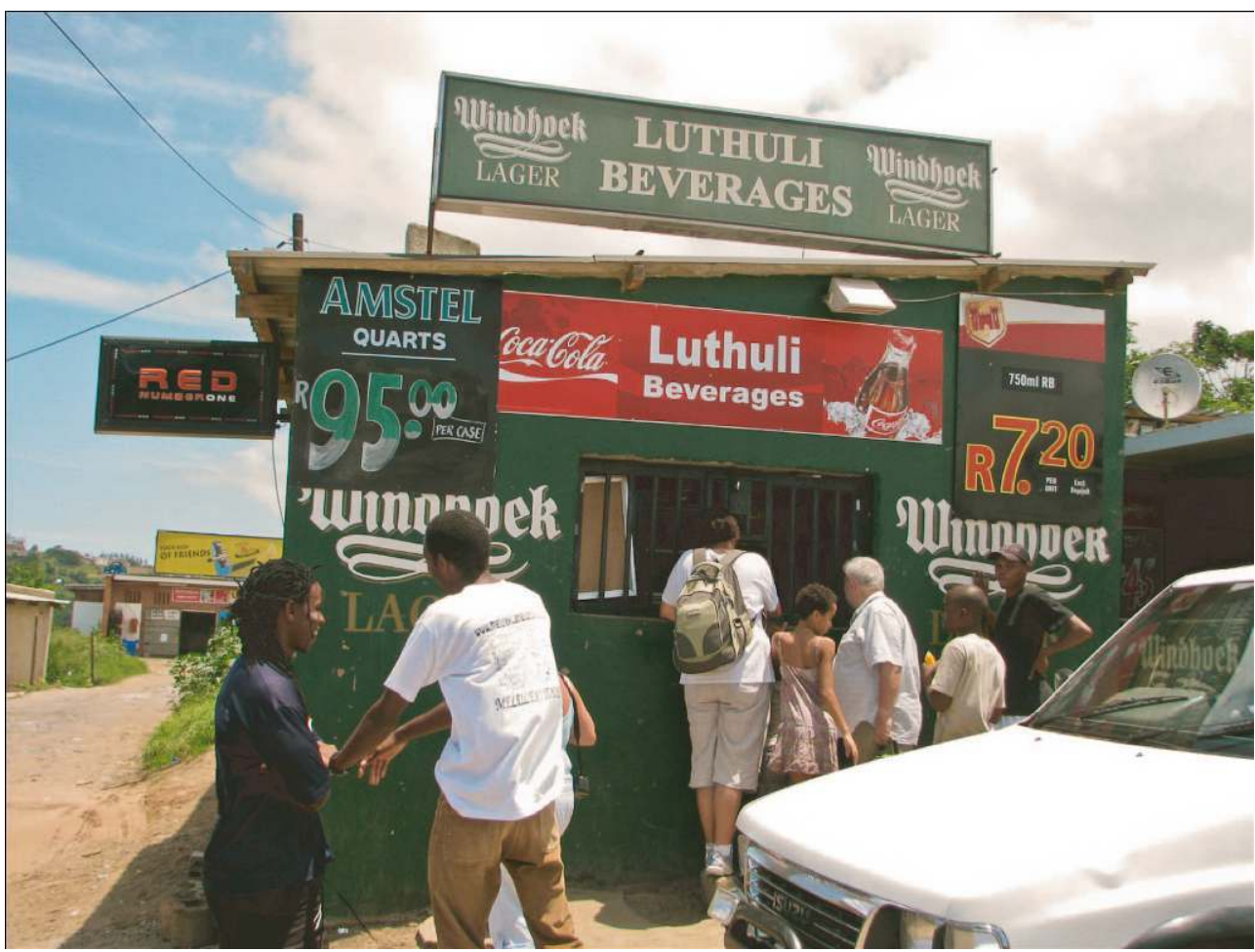

La visite tient lieu de passage obligé lors du tour touristique.

Auteur : Fabrice Folio.

\section{Des Township tours à la Freedom Route : une prestation territoriale réorientée vers la vente d'icônes et de fragments d'histoire}

\section{Viabilité et impact (socio)-économique : KwaZulu-Natal versus Gauteng \& Western Cape?}

Le succès économique des Townships tours est moins marqué au KwaZulu-Natal qu'au Cap ou à Johannesburg (qui abrite, entre autres, le célèbre South Western Township). Ce n'est pas véritablement la qualité de la prestation qui fait défaut. Il est vrai que dans le cadre d'un tour de township, en dehors du minibus équipé, le touriste ne se voit pas proposer des services haut de gamme auxquels il pourrait être habitué, tant dans la restauration, le choix de l'artisanat ou dans la simple disposition de distributeurs de billets et de toilettes publiques (Vaughan, 1999). Toutefois, c'est aussi le cas des autres Township tours au plan national: l'expérience est ailleurs et oblige le visiteur à s'adapter. En d'autres sites, particulièrement à Soweto, plus grand township du pays, les Township tours rencontrent une belle popularité. Selon un rapport du Gauteng Tourism Authority (2002), le nombre de visiteurs accédant à l'unique site Memorial Hector Peterson (du nom du premier écolier mort dans le quartier lors de la célèbre manifestation de 1976) est de 1498 par mois. La compagnie johannesbourgeoise Jimmy's Face to Face Tours (la première à avoir proposé ce type de tours en 1985) est aujourd'hui l'un des plus grands tours opérateurs sur le 
marché. Elle faisait état en 2003 du transport de 3000 touristes par mois environ; de plus petits prestataires évoquent eux une activité basée sur le millier de visiteurs (Ramchander, 2004). En comparaison le Township tour d'Umlazi, plus grand township de Durban et deuxième du pays, accueille 150 visiteurs chaque année (par Meluleki Tours), celui de Sobantu à Pietermaritzburg tout juste 50 à $60^{\circ}$. On saisit à ce moment que ce qui fait la différence est l'ancienneté du tour mais aussi la notoriété associée aux sites. Soweto symbolise la lutte anti-apartheid ${ }^{9}$. Son excursion propose en outre la visite des lieux de résidence de Nelson Mandela et de Desmond Tutu (qui incarnent des must see). Le KwaZulu-Natal se voit qui plus est dépassé par la province du Western Cape, où les tours de townships dans les Cape Flats et surtout à Langa, plus vieux township planifié de Cape Town, sont très prisés ${ }^{10}$. A ce niveau, ce n'est pas tant la réputation historique qui s'exprime qu'une réalité structurelle implacable: le Cap de l'Ouestest la province accueillant le plus de touristes étrangers ultra-marins, mécaniquement ces derniers auront tendance à y expérimenter un tour de township, qu'ils ne renouvelleront pas forcément durant leur séjour (l'idée étant que tous les townships se ressemblent peu ou prou....). Face à ces réalités tangibles, les autorités provinciales estiment tout de même que le KwaZulu-Natal a une carte à jouer, eu égard à la place importante qu'il prend dans le secteur du tourisme d'affaire ou de congrès (articulé sur le International Convention Center de Durban). Dans ce contexte, elles se rendent à l'évidence que les Township tours du KwaZulu-Natal souffrent, notamment par rapport à Johannesburg, de l'absence de « figures iconiques » pour les personnaliser idéalement.

En ce qui concerne le volet participatif et redistributif des Township tours, les données demeurent fragmentaires. Il reste ardu de cerner précisément leur impact positif (Allen \& al, 1988, Brunt \& Courtney, 1999). Nous pouvons il est vrai avancer, à la suite d'Ashley \& al (2000), que le socio-tourisme est parmi les rares sous-secteurs à explicitement prendre en considération les préoccupations des plus pauvres. Goudie \& al (1999) vont dans le même sens lorsqu'ils arguent qu'exclure les townships et les aires informelles de l'agenda des tours opérateurs en Afrique du Sud ne peut que les écarter davantage de la vie sociale et économique du pays et ainsi renforcer les inégalités du passé. Toutefois, dès lors que les tours opérateurs sont souvent tenus par des acteurs extérieurs (où même les guides recrutés sont parfois toujours blancs, Mackay, 2005) et, on l'a vu, les interactions assez superficielles, on peut avec prudence émettre des réserves sur la nature de leurs retombés économiques. Si au tout début, les excursions ont été proposées par les résidents mêmes, les grands tours opérateurs se sont progressivement appropriés ce marché lucratif, s'imposant tant sur le plan du marketing que sur la qualité de l'offre de transport. A cela il faut ajouter le fait que les Licences demeurent difficilement accessibles pour des locaux en manque de qualification (Rolfes \& all, 2009). Butler (1999) rappelle que, d'une part, les prestataires se paient la part du lion de l'excursion, le tarif étant fixé en amont et réglé via l'office du tourisme. D'autre part, que les dépenses internes, générées par les touristes, demeurent assez réduites (achat de produits de consommation ou d'artisanat) et cantonnées à des sections de routes ${ }^{11}$. Ce constat vaut-il prioritairement pour les tours quasi « à la chaîne » de Johannesburg ou du Cap ?

29 En réalité, le constat est à nuancer au plan national, entre les compagnies mêmes. Il revient au touriste d'être attentif. Il s'avère en premier lieu utile de rappeler que le potentiel de développement des Township tours est selon nous indéniable, bien qu'inexploité. L'essor touristique, au cœur de bassins de vie où les défis sont patents, peut conduire à l'établissement d'aménagements structurants, en lien avec les élus locaux et le 
monde associatif. Par effet de gamme, il peut tirer des filières proches, dans la restauration, le commerce ou les services (Scheyvens, 2002a). En second lieu et concernant précisément la participation communautaire des Township tours du KwaZuluNatal, elle serait évaluée comme meilleure en cette contrée que par exemple dans le Gauteng (où plusieurs opérateurs exogènes à la vie locale occupent le terrain, KZN Township Tourism Study, 2008). Dans la province, on retrouve encore souvent de petites structures, parties de la base à l'initiative de passionnés de la vie de « leur » township. Ces derniers se sont rapprochés des organismes touristiques provinciaux pour obtenir leur accréditation $^{12}$. De fait, les structures sont pour le moment plus intimistes et moins professionnelles. Mais, à n'en pas douter, cette démarche va dans le sens de l'ardue politique étatique du Black empowerment, visant à la consolidation d'un entreprenariat noir.

Certes, il convient encore localement d'améliorer la qualité de l'approche et d'y renforcer la participation économique. Priorité durant le tour devrait être donnée, par exemple, aux initiatives conduisant à l'amélioration du cadre de vie des résidents. Car il est oublié trop souvent que là où règne la pauvreté existent en général des gens ou des organisations impliqués chaque jour dans des projets pour la combattre (Hartwell, 2008) : ces acteurs, désireux d'offrir une image plus positive des townships, mériteraient d'être davantage associés aux parcours des Township tours (comme dans le Mamelodi Project à Pretoria). Les autorités provinciales devraient également encourager les hébergements touristiques à s'ouvrir à l'intérieur des townships; cela permettrait aux visiteurs d'y passer plus de temps et d'expérimenter leur vie nocturne. Devant l'hétérogénéité des structures économiques dans ces quartiers, une association du tourisme local (LTA) devrait voir le jour ainsi que des offices du tourisme dans chaque township (KZN Township Tourism Study, 2008). Les compagnies, une fois viables (ce qui ne doit pas exclure un partenariat équilibré à trouver entre les locaux et des tours opérateurs tels que Thompsons), pourraient être invitées à reverser une part de leur profit aux communautés - et il devrait scrupuleusement être indiqué aux touristes celles qui le font. Il a été démontré qu'une relation étroite existait entre les bénéfices touristiques perçus localement et le support de l'activité par les habitants (Pizam \& Milman, 1986, McCool \& Martin, 1994). Enfin, des gestes fondamentaux pourraient être rappelés aux hôtes, dans le cadre d'une charte de bonne conduite établie par les acteurs du secteur ${ }^{13}$. Reste la question d'une meilleure fréquentation de ces tours (qui, parfois, seront la seconde visite d'un township au KwaZulu-Natal), ce qui induit plus de visibilité et un positionnement à réorienter.

\section{La « Route de la liberté » ou les icônes mises en avant : une inflexion récente}

31 C'est dans ce contexte que les autorités du KwaZulu-Natal ont lancé depuis peu la Freedom Route (illustration 6). Cette dernière propose à présent au touriste des visites de sites dans le cadre d'une expérience multiculturelle, périurbaine comme urbaine, chargée d'histoire. Initiée par divers acteurs de l'industrie du tourisme provincial (Tourism KwaZulu-Natal, l'institution publique qui promeut le Zulu Kingdom, plusieurs structures locales ainsi que la compagnie privée de transport African Link Tours), elle cherche à exalter le sentiment de liberté et de lutte attaché à cet idéal, en s'appuyant pour ce faire sur des personnalités illustres. Ces dernières, présentées comme des «icones", 
détiennent toutes une parcelle d'histoire qui a contribué à forger celle de la province. Mais elles disposent d'une aura qui dépasse dorénavant le seul cadre géographique et administratif du KwaZulu-Natal. En conséquence, l'objectif visé est de capitaliser sur le sentiment inaltérable d'espoir ayant succédé à l'ère d'apartheid (dans le prolongement national). En même temps, il s'agit de se focaliser, à un niveau régional, sur l'histoire du KwaZulu-Natal et le parcours d'hommes dont le combat ou le rôle a fini par avoir une influence sur l'ensemble du pays. Afin de capter l'attention du visiteur ayant déjà arpenté Johannesburg ou Cape Town, il lui est ainsi proposé, en relation avec le passif de ces acteurs, de mesurer l'influence notable qu'a pu avoir - à travers eux - cette région sur toute l'Afrique du Sud (et même au delà, comme on le verra).

Illustration 6 - Première de couverture et avant dernière page du prospectus décrivant la Freedom route

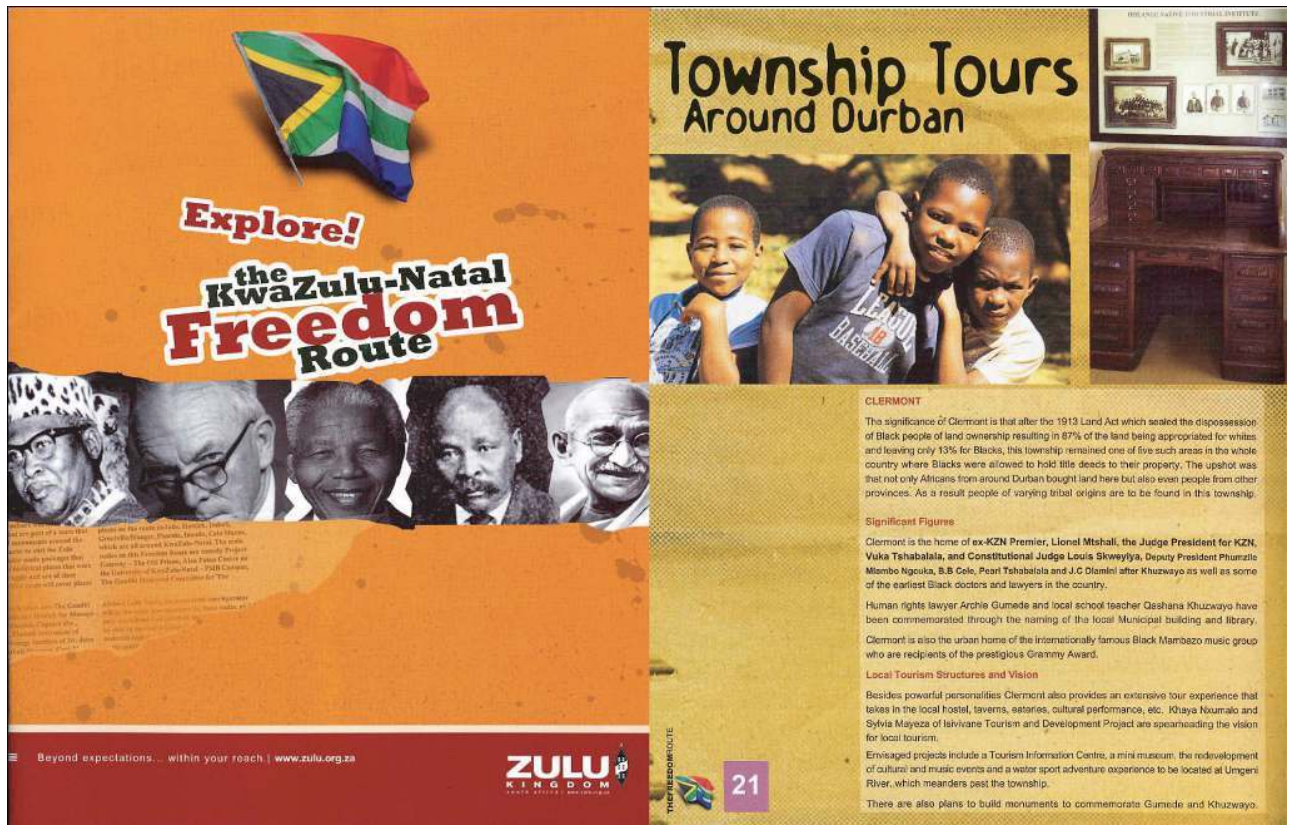

Les icônes historiques (inter)nationales, à la forte empreinte régionale, sont mises en avant, dans une subtile osmose avec le profil dynamique et accueillant des habitants des quartiers périphériques.

Une autre caractéristique de la Freedom Route repose sur le fait que, pour la première fois, les acteurs bénéficient d'une visibilité. De même, il est tenté de les faire travailler en réseau, à travers un affichage cohérent. Le prospectus principal (accessible entre autres au Tourism Junction de Durban) peut en témoigner: il s'agit d'un livret assez bien documenté sur les prestations et compagnies disponibles. Autre trait de particularité, d'un point de vue communautaire et à degré moindre géographique, l'offre revêt une belle diversité. On décloisonne enfin les seuls townships noirs au profit de sites ou de monuments présents dans les espaces centraux voire périurbains, ou encore à la faveur d'autres cultures provinciales. Par exemple la Inanda Heritage Route est sans conteste l'excursion la plus complète et intéressante du KwaZulu-Natal. A travers ce quartier d'implantation spontanée sur site viabilisé, elle propose au visiteur un regard sur des populations contrastées et un aperçu de la richesse des aires périphériques des villes provinciales (via une association d'habitat informel, quoique toléré et de bidonvilles sur terre publique ou coutumière $)^{14}$. A cela s'ajoute un mélange de culture africaine et indienne, Inanda - et plus précisément le Phoenix Settlement - ayant abrité cette 
importante communauté par le passé (et ce en dépit de heurts intercommunautaires), notamment la maison de presse d'un jeune avocat, Mohandas Karamchand Gandhi. Les icônes s'invitent à ce stade : outre la maison de presse rénovée de Gandhi (où il publia son journal engagé : The Opinion - illustration 7), sur le Ohlange Institute, où Nelson Mandela déposa son bulletin de vote en 1994, plane l'ombre de John Langalibalele Dube. Ce dernier fut le premier président du futur African National Congress (ANC, au pouvoir depuis la fin de l'apartheid). En 1901, il fonda et anima ici un institut éducationnel africain, sans équivalent dans toute l'Afrique australe. Pour compléter la liste, le quartier d'Ebuhleni fait découvrir le culte baptiste de l'église de Shembe (la plus large du KwaZulu-Natal, avec deux millions de fidèles) et le parcours de son philosophe et prophète Isaiah Shembe. Autre exemple illustrant cet alliage «icônes-faits historiques-sites pluriels»: à Pietermaritzburg, c'est à un véritable circuit urbain, central et péricentral, que nous sommes conviés, articulé une nouvelle fois sur Gandhi et les lieux fameux qui l'associent à la capitale administrative provinciale ${ }^{15}$. Vers Howick, petite localité au cachet britannique en périphérie de Pietermaritzburg, emmitouflée dans des paysages empreints de culture anglo-saxonne, un Heritage trail inclut, outre la visite du township de Mpophomeni, un Capture site; situé sur la R 103, la stèle cherche à attirer l'attention sur un épisode de l'histoire sud-africaine: il s'agit du lieu où Nelson Rolihlahla Mandela, habillé en chauffeur et de retour de Durban, fut appréhendé par la police en août 1962. Terminons ce tour d'horizon par les musées liés à l'histoire d'oppression et qui trouvent aussi leur place dans la route de la liberté. Aux côtés du KwaMuhle à Durban, le Luthuli Museum à Groutville (plus au nord, sur la R 102, non loin de Stanger), s'articule quant à lui sur un personnage central, associé volontiers à Kwame Nkrumah ou à Martin Luther King: il s'agit du Chief Albert John Mvumbi Luthuli (John Luthuli), fondateur de l'ANC et premier prix Nobel de la paix sud-africain en 1960. 


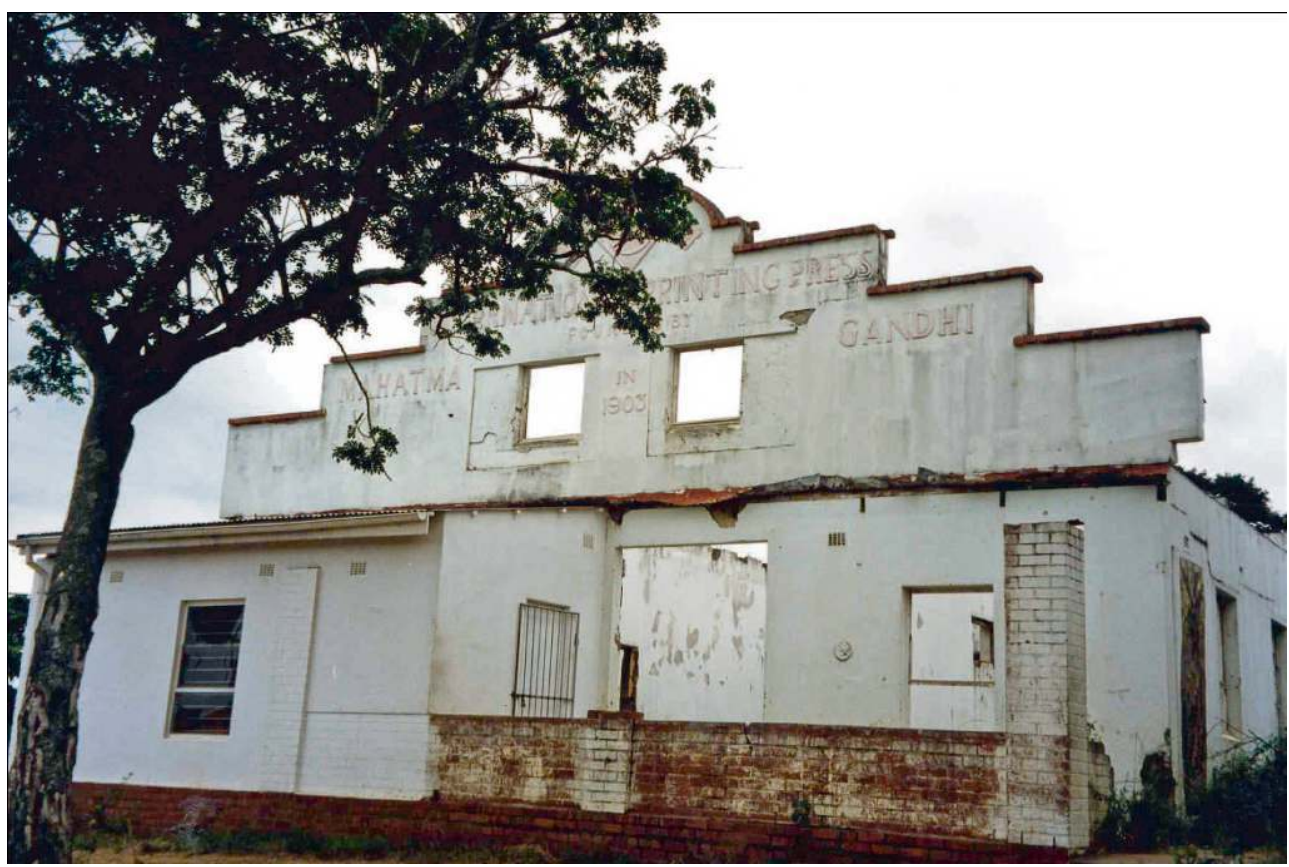

C'est le est le lieu où Gandhi s'établit au nord de Durban durant ses années de formation en Afrique du Sud. II fut détruit par les émeutes interethniques d'Inanda en 1985, faisant suite à l'installation informelle d'une communauté africaine qui l'a rebaptisé Bhambayi. A la fin des années 1990, la zone fut restaurée et partiellement reconstruite, devenant ainsi un Heritage site (au fort symbole de réconciliation) et une attraction touristique en février 2000

\section{Illustration $7 \mathrm{~b}$ - Phoenix Settlement restauré}

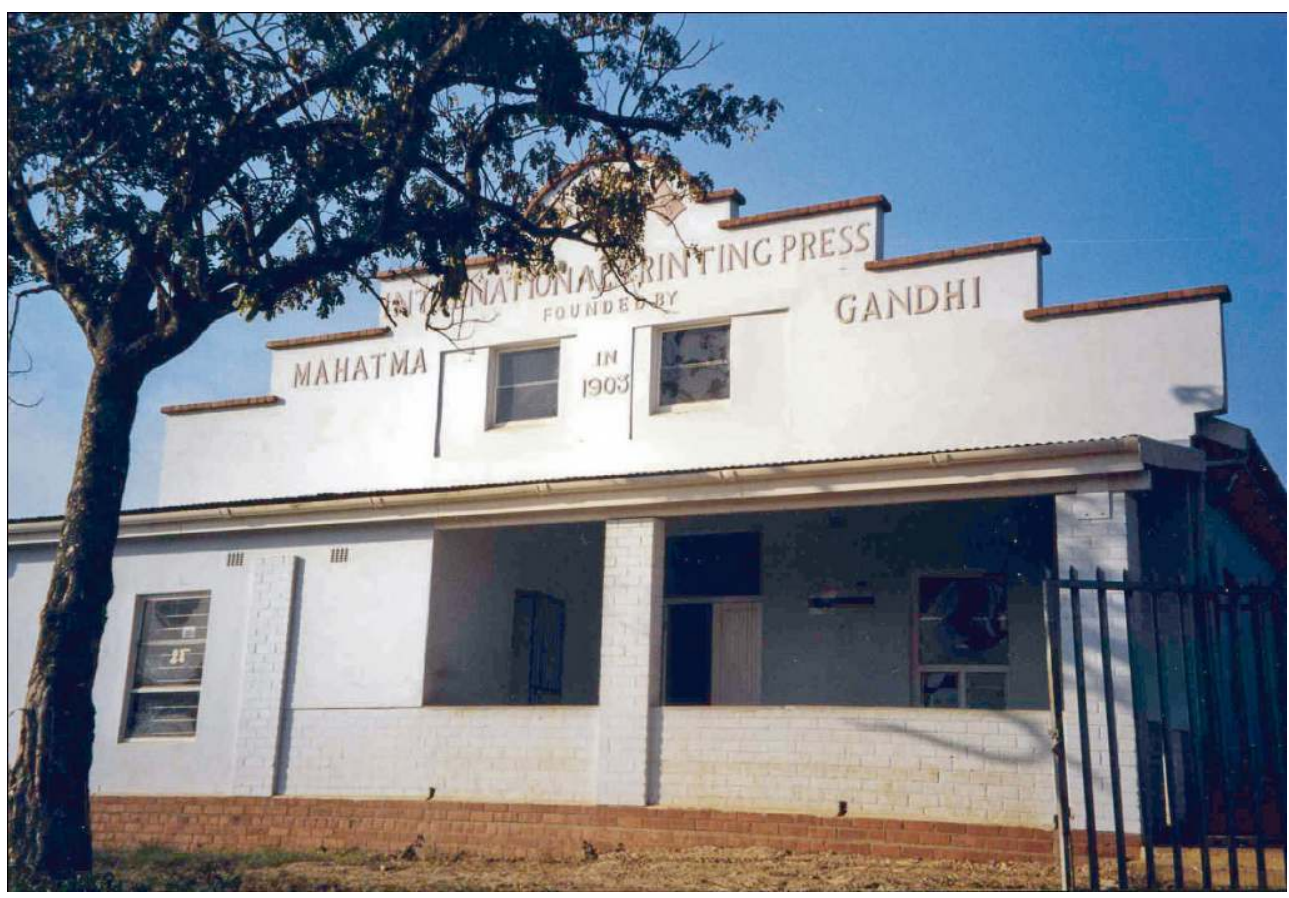

Auteur : Fabrice Folio. 
En somme, la priorité des édiles touristiques régionaux est d'afficher de la lisibilité et plus de connexions dans l'offre mémorielle en lien avec l'apartheid. Ils aspirent à le concrétiser en promouvant des icônes devenues nationales (voire mondiales), dont l'implication sur place a été décisive, parfois par le biais d'actes précurseurs ou annonciateurs. En revenant à notre essai de définition vu en partie 1, on comprend que la part pédagogique de l'expérience tend à être valorisée, dans une recherche d'équilibre avec le versant sensationnel, mais sans pour autant délaisser le registre émotionnel. D'ailleurs, les tours de townships conventionnels présents dans la Freedom Route tendent à esquisser l'originalité du KwaZulu-Natal, replacée sur un plan national. Ainsi, la visite d'Edendale/Imbali à Pietermaritzburg accorde une place aux victimes et aux lieux des émeutes opposant l'IFP et l'ANC dans les années 1990 (et qui mirent en péril l'avènement démocratique national qui se profilait). Les ex-quartiers noirs peuvent aussi, et c'est là rafraîchissant, être abordés sous un angle culturel valorisant. Par exemple, Clermont est l'un des cinq townships municipaux dans le pays où les Africains étaient autorisés à détenir un titre de propriété. A présent, il est cité comme le lieu de résidence du plus prestigieux groupe vocal sud-africain, représentatif du style musical isicathamiya: Ladysmith Black Mambazo (originaire par ailleurs de la région). L'expérience s'approche un peu plus de ce que Bartis (1998) intitule un "tourisme d'héritage noir ", à ceci près que la Freedom Route est sensiblement moins afro-centrée et davantage multiculturelle. Tout cela suffira-t-il à faire de ce segment touristique un incontournable de la province?

\section{Une offre inaboutie ou comment relier l'infra-urbain, le multiculturel et un passé pionnier}

34 Si cette offre a le mérite de la clarté et d'une assise culturelle, il n'est pourtant pas certain qu'elle parvienne véritablement à s'imposer comme un créneau phare du KwaZulu-Natal. La province risque-t-elle encore de continuer à s'appuyer sur son potentiel balnéaire, sportif et ses réserves naturelles? Articuler sa prestation de Township tours sur des personnalités ou des icônes est dans les faits déjà pratiqué par Soweto. En outre, l'idéal de lutte pour la liberté, message phare de la nation arc-en-ciel, ne peut s'émanciper de l'Afrique du Sud toute entière (et de ses ambitions universalistes) et, par la même, prétendre à l'originalité. Tout se passe comme si le KwaZulu-Natal tente au mieux de tirer son épingle du jeu en (re)captant une fraction de la clientèle attirée par ce type de prestation. Mais la ville du Cap, par sa position de plaque tournante des visiteurs ultramarins, ainsi que Johannesburg, véritable bouillon urbain historique, garderont, sur ces aspects précis, une longueur d'avance. Finalement, l'offre des Township tours du KwaZuluNatal, même si évoluant dans le bon sens, demeure à notre avis quelque peu timide, alors qu'elle dispose d'une base de renouvellement plus forte.

En premier lieu, le contenu de la Freedom Route reste inachevé, que ce soit les éléments dessinant un multiculturalisme prudent comme ceux ébauchant des faits historiques plutôt sélectifs. En dépit d'un éclairage jeté sur l'héritage de personnages émanant de la communauté indienne (Ghandi) ou blanche (le romancier Alan Paton, auteur de Cry, the Boloved Country et natif du KwaZulu-Natal), il est évident que les lieux de résidence des populations autres que noires persistent à demeurer à l'écart des Township tours. Rappelons à ce stade, pour prévenir toute remarque sur le caractère a priori singulier de l'activité, que les townships, issus de la partition spatiale mono-raciale, ont naguère été attribués à l'ensemble des communautés non-blanches, soit noire mais aussi indienne et 
coloured. A chacune d'entre-elles correspondaient un espace propre, un bâti spécifique et des niveaux donnés de services publics et d'équipements. Ne serait-il pas intéressant de leur accorder un intérêt qui irait plus loin que le seul souci de l'équité touristique, en particulier dans une province connue tant pour son peuple zoulou que pour son identité indienne (plus forte diaspora au monde établie en dehors de l'Inde) ? Le but serait de permettre d'en savoir davantage sur l'hétérogénéité initiale du projet de développement séparé, cumulée aux évolutions internes qu'ont ensuite pu connaître ces quartiers. Certes, il existe bien à Durban les Merewent \& Wentworth Coloured Townships Tour ainsi que la découverte du quartier indien de Chatsworth (où trône le majestueux «Temple de la connaissance »). Pourtant ces derniers ont une faible représentativité dans la Freedom Route. Or, afin de saisir dans son intégralité l'héritage urbain de l'apartheid, il conviendrait de jouer sur le couple ville visible/invisible, en donnant la place qu'elles méritent à toutes les communautés (et à leur apport identitaire). Il peut ainsi être possible d'envisager une "route de l'apartheid urbain" qui traverserait l'espace compartimenté de la ville, de l'hyper-centre jusqu'à son ultime couronne. Les territoires dits blancs, indiens et coloured, la façon dont ils ont été conçus, leur patrimoine architectural et leur recomposition, bénéficieraient à ce moment d'un regard nouveau, mis en perspective avec les townships noirs.

Dans ce prolongement, malgré les évènements historiques intéressants sur lesquels s'attarde la Freedom Route, il nous semble que l'on pourrait aller plus loin, en ne se focalisant pas uniquement sur des icônes. A tout point de vue, le côté avant-gardiste du KwaZulu-Natal est incontestable et mérite qu'on s'y attarde. Les mesures de restrictions commerciales et de contrôles des activités indiennes en bordure du centre-ville de Durban ont très tôt conduit à la ségrégation foncière et résidentielle (Mainet-Valleix, 2002). C'est dans la province qu'ont ainsi vu le jour les premières Indian locations en 1871, préfigurant les quartiers ségrégués planifiés. De même, le togt labour system, ancêtre de l' influx control (et donc de la régulation de la mobilité des Noirs en ville), à l'attention des ouvriers temporaires, est apparu localement dès 1874 . Ces derniers, via un système de permis, résidaient en des barracks insalubres qui inspireront plus tard les hostels des townships. Le Durban System visait à assurer, entre 1909 et 1930, l'administration des Africains par un monopole municipal sur la vente de la bière ; il deviendra un modèle du genre de gestion financièrement déléguée. Le passé pionnier du KwaZulu-Natal peut ainsi devenir un argument de vente. De même, dans le cadre du Shepstone System, les premières réserves africaines, antérieures aux bantoustans, sont nées ici : cela mériterait d'être explicité dans le sens où certains townships noirs, érigés à la suite du Grand apartheid dans les années 1970, ont été établis sur ces zones rurales (ex-Kwazulu), à mode de chefferie coutumière. Cela nous amène finalement à penser que les Township tours du KwaZulu-Natal ne peuvent laisser à l'écart les zones tribales avoisinant ses espaces urbains (ce qu'effectuent déjà Inanda ou Mpophomeni mais le discours n'est pas vraiment orienté en ce sens). Il n'y a qu'à voir l'interrelation entre les aires rurales denses, les zones informelles et enfin l'habitat planifié des townships, lieux des «évadés (Guyot, 2005), en périphérie des villes de Durban, de Pietermaritzburg ou de Richards Bay. D'une excursion culturelle exclusivement urbaine, le « tour intégral » prendrait alors des allures de cheminement en pénétrante, depuis la ville-centre - en y incluant les muthi market ou marchés deremèdes traditionnels - jusqu'à ses aires de fonctionnement inter-reliées périurbaines et semi-rurales. Dans l'absolu, cela pourrait rendre possible le raccordement des Township tours à certains villages culturels (la plupart étant implantés à proximité relative des villes). Ce qui aurait alors le mérite de profiter de l'attrait notoire des ultra- 
marins pour la culture zouloue, dont le référent, au sein de l'Afrique du Sud contemporaine et bien au-delà, est omniprésent (Giraut, Boujrouf, Rey, 2010). Evidemment, combiner le legs urbain de l'apartheid, en des quartiers expressifs, et celui des villages culturels, au caractère parfois fixiste et artificiel, serait un paradoxe (encore qu'il permettrait aux touristes de relativiser cette dernière attraction, en prenant en considération des zones d'habitat « réelles » limitrophes, en perpétuelle évolution). Mais même s'ils demeurent pour le moment moins vendus par les tours opérateurs, n'oublions pas qu'existent et que gagnent à être connus des villages culturels touristiques plus autonomes que PheZulu ou Shakaland au KwaZulu-Natal, tels Ecabazini ou Nanoti Valley (Marschall, 2003); appropriés par la communauté locale (qui a toute latitude sur la scénarisation du tour), ils s'évertuent à montrer une culture rurale dynamique.

\section{Conclusion}

Les Township tours sont des attractions touristiques à présent installées dans le paysage urbain du KwaZulu-Natal et plus sûrement de l'Afrique du Sud. Il s'agit d'une nouvelle niche touristique au sein d'un secteur en constante mutation et confronté à une clientèle exigeante, en attente de nouvelles expériences. Dans un contexte de mondialisation démultipliant les flux touristiques et aggravant tout à la fois les inégalités de richesse, des sous-espaces mondiaux incarnant le « Sud » s'ouvrent à des visiteurs du « Nord », dans un esprit singulier de curiosité, de désir de compréhension et de solidarité de contact. En Afrique australe, les Township tours ne concernent d'ailleurs pas que l'Afrique du Sud: le township namibien de Katutura propose à titre d'exemple ce type de circuit. Leur originalité est de réaffirmer un épisode phare d'une histoire régionale brutale et refoulée. Qui plus est, ils mettent en avant la composante majoritaire de la population ici sudafricaine, laissant de facto au visiteur une impression de juste retour des choses. Par delà les interrogations et réserves sur la nature des interactions, l'apport culturel et surtout le volet participatif, ce segment touristique multi-sensoriel vise aussi à être bien organisé pour se pérenniser. Effectivement, si les touristes disent désirer l'originalité, l'authenticité et de plus en plus les émotions fortes (Craik, 1997), ils sollicitent également une expérience négociée au sein de la bulle touristique, soit un environnement ludique et pédagogique, à sensations mais sécurisé, où ils deviennent des acteurs guidés et avisés. Nous aspirons toutefois à dépasser le débat éthique quant au voyeurisme (non pas que ce biais soit absent, loin s'en faut, de certaines des excursions). La visite de quartiers " ethniques » se pratique aujourd'hui en d'autres lieux (à Singapour, entre Chinatown, Arab street et Little India ou à Londres avec Banglatown), sans que l'on ne s'en émeuve outre mesure : cela incite à considérer la question non pas en des termes identitaires et d'espaces vécus, mais bien sous l'angle du choc des extrêmes de richesse, de l'approche éminemment sensible entre privilégiés et démunis. La pauvreté ne peut que malaisément devenir esthétique et instrument de marchandisation (Mowforth \& Munt, 1998). Or, à l'heure actuelle, les tours disponibles en Afrique du Sud restent, entre eux, très disparates. Il est vrai qu'un des paradoxes du tourisme pro-pauvre est qu'en annihilant cette dernière, il perdrait sa raison d'être. C'est pour cette raison que miser sur l'axe patrimonial et pédagogique des Township tours semble la voie étroite recherchée. Ce sociotourisme devient à l'image de l'éco-tourisme, dans ses espoirs suscités comme dans la réalité du terrain : de grands principes de base de durabilité quoiqu'appliqués de façon parcellaire in situ, où les insuffisances voire les dérives ne sont pas loin. Quoi qu'il en soit, 
si les Township tours sont désirés par les espaces en déshérence et que - ne serait ce qu'une partie - de la communauté locale, instigatrice, y devient partie véritablement prenante, il serait assez spécieux de culpabiliser des visiteurs démontrant un intérêt finalement humain pour la face économiquement pauvre mais culturellement intense de ce pays. Surtout que cette dernière mériterait de s'inscrire dans un cadre spatial et communautaire plus vaste, en particulier au KwaZulu-Natal. La profondeur du champ historique et le potentiel d'une mise en tourisme alternatif y sont en effet avérés.

\section{BIBLIOGRAPHIE}

Allemand S., Dagorn R-E., Vilaça O., 2005. La géographie contemporaine. Collection Idées reçues, Le Cavalier Bleu, $126 \mathrm{p}$.

Allen L, Long P.R. \& Kieselbach S., 1988. The impacts of tourism development on residents' perceptions of community life. Journal of Travel Research, 27 (1), p 16-21.

Ashley C., Boyd C., \& Goodwin H., 2000. Pro-poor tourism: Putting poverty at the heart of the tourism agenda. Natural Resource Perspectives, Overseas Development Institute, London, 51, p 1-12.

Ashley C., Roe D., Goodwin H., 2001. Pro-poor tourism strategies: making tourism work for the poor: a review of experience. Pro-Poor Tourism Report 1, Overseas Development Institute/ International Institute and Development/CRT, The Russel Press, London.

Bartis H., 1998, A national black heritage trail in the Eastern Cape Province, South Africa: Is it an option? In Hall D., \& O'Hanlon L., (eds), Rural tourism management: sustainable options, conference proceedings, 9-12 September, Scottish Agriculture College, Auchincruive, p 17-28.

Brunt P., Courtney P., 1999. Host perceptions and socio-cultural impacts. Annals of Tourism Research, 26 (3), p 493-515.

Butler S.R, 2003a. Entrepreneurial Heritage: The Townships as Exhibition in Post-Apartheid Cape Town. U npublished paper.

Butler S.R., 2003b. The Museum, The Tour, the Senses. Department of Anthropology and Sociology, UBC, paper prepared for the CONSERT Meeting, November 13, $12 \mathrm{p}$.

Butler S.R., 1999. Townships Tours: packaging the new South Africa. Southern Africa Report, Vol 14, $\mathrm{N}^{\circ} 4, \mathrm{p}$ 24-30.

Chapman K., 2003. Township tours offer glimpe of 'Mandela's country', http://www.cnn.com/ SPECIALS/1999/safrican.elections/stories/township.tours/

DACT, 2008. White Paper on the Development and Promotion of Tourism in KwaZulu-Natal, Government of KwaZulu-Natal. Department of Arts, Culture and Tourism.

Dondolo L., 2001. Depicting history at Sivuyile Township Tourism Center. Paper presented at the Mapping Alternatives: Debating New Heritage Practices Conference, University of Cape Town, 26-27 Septembre.

Dubin S, C., 2006. Transforming Museums: Mounting Queen Victoria in a Democratic South Africa. New York: Palmgrave Macmillan, 339 p. 
Else D., Murray J., \& Swaney D., 1997. Africa - the south. Lonely Planet, Melbourne.

Folio F., 2010, Tourisme culturel au KwaZulu-Natal, Afrique du Sud : d'une réhabilitation historique à des produits touristiques controversés. In Jauze, J-M., Combeau, Y. (dir.), Géographes et Historiens dans la compréhension et la gouvernance des espaces et sociétés de l'océan Indien, «Terres et sociétés indocéaniques ", Université de La Réunion, p. 61-82.

Fabrice Folio et Anaëlle Derroisne, 2010. Ecotourisme et parcs naturels : innovations et contradictions sud-africaines, le cas de HIP (Hluhluwe-Imfolozi Park). EchoGéo [En ligne], numéro 13 | 2010, mis en ligne le 20 septembre 2010. http://echogeo.revues.org/11769

Folio F., 2007. Construction géohistorique du KwaZulu-Natal (Afrique du Sud). EspacesTemps.net, Textuel, 01.09.2007, http://espacestemps.net/document3191.html

Folio F., 2004. Villes post-apartheid au Kwazulu-Natal : une déclinaison du modèle de Davies. L'Information géographique, Vol. 68, no 4, p. 320-339.

Francis S., Dugmore H., \& Rico, 2002 (1994 pour la première édition). Free at Last. Madam \& Eve, Penguin Book, South Africa, 176 p.

Freire-Mederios B., 2009. The Favela and Its Touristic Transits. Geoforum, 2009.

Gentleman A., 2006. "Slum tours: a day trip too far?" The Observer, May, 7, 2006.

Gervais-Lambony P., 2009. L'Afrique du Sud. Collection Idées reçues, Le Cavalier Bleu, 127 p. Gervais-Lambony P., 2001. Petite histoire d'espace et d'identité dans une ville sud-africaine. Champs Psychosomatiques, $\mathrm{n}^{\circ}$ 21, p. 119-131.

Giraut F., Boujrouf S., Rey B., 2010. Le référent zoulou omniprésent, EchoGéo, numéro 13, juin 2010/août 2010. http://echogeo.revues.org/12039

Goudie S.C., Khan F., \& Kilian D., 1999. Transforming tourism: Black empowerment, heritage and identity beyond apartheid. South African Geographical Journal, 81 (1), p. 22-31.

Guyot S., 2005. Essai de sociologie territoriale sud-africaine. EspacesTemps.net, Textuel, 30.04.2005. http://espacestemps.net/document1317.html

Hartwell L., 2008. Two Different Approaches to Township Tours. Political Sciences Department at the University of Pretoria, The Mamelodi Project http://blog.mamelodiproject.org/2008/06/twodifferent-approaches-to-township-tours/

Hernandez J., 2008. Le tourisme macabre à la Nouvelle-Orléans après Katrina : résilience et mémorialisation des espaces affectés par des catastrophes majeures. Norois, 3 ( $\left.\mathrm{n}^{\circ} 208\right)$.

Hewison R., 1987. The Heritage industry : Britain in a climate of decline. London, Methuen.

Holden P., 1984. Alternative tourism. Report of Workshop on Alternative Tourism with a focus on Asia, Ecumenical Council on Third World Tourism, Chiang Mai, 26 April-8 May.

Holliger J., 2005, Quelles possibilités de développement afin de combler les fosses entre populations sudafricaines, Diplôme de Gestionnaire en Tourisme de l'Ecole Suisse de Tourisme de Sierre.

Jeuwsen B., 1996. Cultural Tourism in Eastern Bushmaland. Notes on a Fieldrip Graduate Programme in Cultural and Media studies, University of Natal, Durban.

Kendle A., 2008. Poverty Tourism: Exploring the Slums of India, Brazil and South Africa, Vagabondish The Travelzine for today's vagabond. http://www.vagabondish.com/povertytourism-touring-the-slums-of-india-brazil-and-south-africa/ 
Kendle A., 2008. Dark Tourism : A Fine Line between Curiosity and Exploitation, Vagabondish The Travelzine for today's vagabond. http://www.vagabondish.com/dark-tourism-travel-tours/

KwaZulu-Natal Township Tourism Study, 2008. Zulu Kingdom, 69 p.

Lamy-Giner M-A., Guébourg J-L., 2005. Le tourisme international en Afrique du Sud, Cybergeo, Espace, Société, Territoire, article 331, mis en ligne le 12 décembre 2005.

Lennon J., Foley M., 2000. Dark Tourism. Cengage Learning Business Press, 194 p.

Mabogane M., Callaghan R., 2002. Swinging Safaris in Soweto, http://www.mg.co.za/mg/africa/ soweto/html

Mackay M., 2005. Township tour safety 'requires black guides. Cape Argus, December 02, 2005, Edition 1. http://www.capeargus.co.za/index.php?fArticleId=3020038

Mainet-Valleix H., 2002. Durban. Les Indiens, leurs territoires, leur identité. Karthala, Collection Hommes et sociétés, $269 \mathrm{p}$.

Marschall S., 2005. Making Money with Memories: The fusion of Heritage, Tourism an Identity Formation in South Africa. Historia, 50 (1), May 2005, p. 103-122.

Marschall S., 2003. Mind the difference : a comparative analysis of Zulu cultural villages in KwaZulu-Natal. Southern African Humanities, Vol. 15, Pietermaritzburg, p. 109-127.

Masland T., Esther P., \& Mike C., 2002. Turning History into tourism, Newsweek (Atlantic edition), 140 (16), p. 42-48.

McCool S.F., Martin S., 1994. Community attachment and attitudes towards tourism development. Journal of Travel Research, 32 (3), p. 29-34.

Mowforth M., \& Munt I., 1998. Tourism and Sustainability: New tourism in the Third World. Routledge, London.

Ndela N.F., 2002. Representations of Zulu Cultural Identity in cultural tourism : a case study of Izintaba Zulu Cultural Village. University of Natal, Durban.

Perkins T.E., 1979. Rethinking stereotypes. In Barrett M., Corrigan P., Kuhn A. \& Wolff J. eds, Ideology and cultural production, London, Croom Helm, p. 135-159.

Pizam A., Milman A., 1986. The social impact of tourism. Tourism recreation research, 11 (1), p. 29-33.

Poon A., 1993. Tourism, technology and competitive strategies, Oxford, CAB. Wallingford.

Ramchander P., 2004. Towards the Responsible Management of the socio-cultural impact o Township tourism. Submitted in partial fulfillment of the requirements for the degree of Philosophiae Doctor in the Faculty of Economic and Management Sciences, University of Pretoria, Department of Tourism Management.

Ramchander P., 2003. Township tourism - blessing or blight : the case of Soweto, Paper presented at the Atlas Expert Cultural Tourism Group Conference on Cultural Tourism: Globalising the Local Localising the Global, Barcelona, 1 November.

Ritzer G., \& Liska A., 1997. 'McDisneyization' and 'post-tourism': complementary perspectives on contempory tourism. Tourism cultures: transformations of travel and theory, Rojek, C., \& Urry, J. eds, London, Routledge, p. 96-109.

Rogerson C., 2006. Pro-Poor local economic development in South Africa: The role of pro-poor tourism. Local Environment, 11 (1), January 2006, p. 37-60. 
Rogerson M., Visser G. Urban Tourism in the Developing World, The South African Experience. London, Transaction Publishers, $337 \mathrm{p}$.

Rolfes M., Steinbrink M., \& Uhl C., 2009. Townships as Attraction, an Empirical Study of Township in Cape Town. Praxis Kultur- und Sozialgeographie, PKS 46, Universitätsverlag Potsdam, 60 p.

Scheyvens R., 2002a. Tourism development: empowering communities. Harlow, Prentice Hall.

Scheyvens R., 2002b. Poverty tourism. Development Bulletin, Massey University, p. 18-21.

Seaton A., V., 1996. Guided by the Dark - from Thanatopis to Thanatourism. International Journal of Heritage Studies, 2(4): p. 234-244.

Stallybrass P., \& Allon W., 1986. The Politics and Poetics of Transgression, Ithaca, Cornell University Press.

Staszak J-F., 2008. Qu'est-ce que l'exotisme, Le Globe - Tome 148, p. 7-30.

Statistic of our Tourism Sector, 2008. Zulu Kingdom.

Tomaselli K.G., 2001. The semiotics of anthropological authenticity: how cultures change to fit their media image. Journal of Natal and Zulu History, 19, p. 63-73.

Tourismes 1 - Lieux Communs, 2002, Equipe MIT, Collection Mappemonde, Belin, 320 p.

Turnbridge J. E., Ashworthn G. J., 1996. Dissonant Heritage - The Management of the Past as a Ressource in Conflict. New York: John Wiley \& Sons, 314 p.

Vaugham A., \& al, 1999. Inanda Tourism Development Business Plan. McIntosh Xaba and Associates.

Wells H., 2000. A critical reflection on cultural tourism in Africa : the power of European imagery. Paper read at the ATLAS Conference: Cultural tourism in Africa: strategies for the new millennium, Mombasa, Kenya, 14-16 December 2000.

Weiner E., 2008. “Slum Visits: Tourism or Voyeurism?”, New York Times - Travel, March, 9, 2008.

Wang C., 2001. Is Pro-poor Tourism viable? Cultural Tourism as sustainable Development in Zulu and Bushman Communities. Master's Project, University of Natal, Graduate Program in Cultural and Media studies.

\section{NOTES}

1. Toutefois, il convient de ne pas occulter le fait que les principaux heurts, dans les décennies 1980-90, ont en priorité concerné les populations des ghettos noirs en interne (bien qu'instrumentalisés par le pouvoir en place), à Soweto comme dans les townships de Durban : ils avaient en effet pour grille de lecture le clivage politique opposant l'ANC et l'UDF - partis panafricains de libération alors interdits - au parti nationaliste Inkhata (IFP) dirigeant le homeland (ou bantoustan : Etat noir fantoche) du KwaZulu. Ce dernier, sur l'air du « diviser pour mieux régner », bénéficiait de l'appui et de la logistique du Parti National afrikaner à la tête du pays.

2. Au total, $59 \%$ des visiteurs ayant quitté le KwaZulu-Natal par voie aérienne en 2007 déclarent avoir expérimenté une activité liée à la culture, à l'histoire et à l'héritage (SAT, 2008). Cela reste certes inférieur aux pratiques relatives à la vie sauvage (70\%), à la balnéarité (73\%) ou encore à la vie nocturne ou au shopping (plus de $90 \%$ ). Toutefois, cette part est en progression chez les visiteurs ultramarins, pour qui ces sous-secteurs représentent des activités d'appoint lors de leur séjour (source : M W. Tifflin, Tourism KZN, 2009).

3. http://www.slate.fr/story/13525/le-tourisme-catastrophe-bresil-est-un-sport-de-combat. 
4. Par là, ils se différencient du Doomsday tourism («jugement dernier »), soit la visite de sites amenés à évoluer sous les effets du dérèglement climatique (Grande barrière de corail, glaciers arctiques ou Mont Kilimandjaro), du Grief Tourism ("peine, chagrin») également appelé Thanatourism (Seaton, 1996), qui est la visite d'un théâtre tragique historique (camps de concentration de Auschwitz, Soham en Angleterre, Ground Zero à New-York ; notons que Robben Island au large du Cap peut aussi être rangé dans cette catégorie) et enfin du Disaster tourism (lieu célèbre d'une catastrophe naturelle), pour lequel on peut citer les exemples du sud-est de l'Asie après le Tsunami de 2004, de la Nouvelle-Orléans après Katrina (Hernandez, 2008) et on se souvient, en 2010, des croisiéristes ayant mouillé au large d'une Haïti meurtrie.

5. Entretien avec Nhlanhla Mthembu (Sobantu Tour) en octobre 2009.

6. Par exemple à travers l'érection de bidonvilles, de backyard shacks - logements de fortune d'arrière-cour - ou encore par l'entremise des maisons ouvrières standardisées, agrandies au fil du temps.

7. Il convient toutefois de distinguer trois sortes de tours (Rolfes \& al, 2009, terrain, 2006-2009) : ceux résolument ancrés dans le legs d'apartheid à la lecture assez figée ; ceux montrant surtout un côté positif et convivial, faisant l'impasse sur ce qui pourrait mettre le touriste mal à l'aise ; enfin ceux montant les townships avec leur part de problèmes et d'inertie mais aussi de changements et de développement. Pour ces derniers, l'hétérogénéité ambiante est de mise, plus qu'un sentiment minimaliste, pour ne pas dire de frustration.

8. Entretiens avec M. Mgobhozi (Meluleki Tours) et Nhlanhla Mthembu (Sobantu Tour) en octobre 2009.

9. Surtout au travers de cette marche des écoliers s'opposant à l'apprentissage de l'afrikaans en 1976, dépeint dans le roman d'André Brink : Une saison blanche et sèche. Au terme de cette journée, la lutte contre l'apartheid va connaître un tournant avec des mobilisations massives voire des émeutes dans la presque totalité des townships du pays, lesquels deviennent des espaces ingouvernables (Mabogane \& Callaghan, 2002).

10. Selon Rolfes \& all (2009), le nombre de visiteurs y est estimé à 300000 par an (contre 40000 dans les favelas tours de Rio), soit $25 \%$ de l'activité touristique totale. Les Township tours y deviennent un phénomène de masse, un Must do au même titre qu'une excursion sur la montage de la Table, à Cape Point ou dans les winelands.

11. Le Mail \& Guardian a ainsi révélé que les tours opérateurs n'offraient l'opportunité de découvrir qu'une quinzaine de sheebens au grand maximum, sur les 5600 que compte le plus vaste township d'Afrique du Sud, au millier de touristes visitant Soweto quotidiennement (année 1998).

12. Entretien avec M W. Tifflin du Tourism KZN (2009).

13. Comme de ne pas multiplier les photographies, préserver l'intimité des individus (bannir l'immixtion dans les logements ou shacks) ou ne pas offrir de cadeaux, de produits alimentaires et encore moins d'argent aux enfants, mais plutôt à des associations caritatives (afin de pas cultiver sur place de quelconque réflexes de charité).

14. C'est aussi le cas du tour de Cato Manor, à ceci près qu'il s'agit pour ce dernier d'un quartier péricentral mixte, interdit sur des bases sanitaires et déclaré zone blanche (à l'instar du District Six) en 1959. Réoccupé ensuite de façon spontanée par de nombreuses familles, il offre depuis un grand flou quant au mode de propriété local. La municipalité d'eThekwini a fait de Cato Manor un de ses symboles d'opération de redéveloppement (à travers des logements sociaux et des équipements publics), ajoutant encore à l'hétérogénéité extrême des lieux.

15. Rappelons que son combat pour les membres de sa communauté, organisé autour du Satyagraha (résistance passive), s'est initié après son éviction forcée d'un train en marche à Pietermaritzburg en 1893. Outre la station de chemin de fer rénovée et commémorant cet événement, dans Church Street Mall (le cœur du centre-ville), trône une imposante statue du Mahatma ; non loin de là, une librairie Gandhi est également en construction. 


\section{RÉSUMÉS}

Des processus de réappropriation identitaire et historique, à finalité économique, sont à l'œuvre à l'heure actuelle en Afrique du Sud. Un type de tourisme au caractère authentique et interactif connaît notamment un certain engouement : les Township tours. Il assume un épisode médiatisé de l'histoire du pays, soit l'apartheid urbain, en tentant d'inverser l'image répulsive qui lui est associée. L'attirance pour les townships se nourrit d'un désir de compréhension de la ségrégation raciale institutionnalisée et de la mise à bas d'une politique inique. Paradoxalement, la réputation de dangerosité, de pauvreté et "d'informalité » accolée à ces quartiers noirs semble aussi agir comme un produit d'appel. A travers l'exemple de la province du KwaZulu-Natal, il sera vu que cette activité socio-touristique s'oriente à présent vers un multiculturalisme prudent, porté par des icônes (inter)nationales, avec en toile de fond les velléités de développement local.

Processes of identity and historical appropriation, rooted in economic purpose, are today arising in South Africa. A tourism activity, based on authenticity and interaction, becomes particularly popular: the Township tours. It assumes an episode of the country history, i.e. urban segregation, which was given a lot of media coverage. In the meantime, it tries to invert the repulsive image that is commonly associated with apartheid. The attraction for township areas has to be linked to the wish of understanding the institutionalized racial discrimination in South Africa, as well as its pacific end. Paradoxically, the reputation of dangerousness, poverty and informality of those former black urban areas seems to act as a loss leader. Through the example of the province of KwaZulu-Natal, it will be seen that this new socio-tourist activity is now playing a multiculturalism card carried by popular icons, with the local development target in the background.

\section{INDEX}

Mots-clés : KwaZulu-Natal, township tour, socio-tourisme, histoire, culture, pauvreté

Keywords : sociotourism, history, poverty

\section{AUTEUR}

\section{FABRICE FOLIO}

Fabrice Folio (fabrice-jm.folio@univ-reunion.fr) est maître de conférences au département de géographie et membre du Centre de Recherches et d'Etudes en Géographie(CREGUR), Université de La Réunion. 